\title{
The Lomax-Claim Model: Bivariate Extension and Applications to Financial Data
}

\author{
Jin Zhao, ${ }^{1}$ Humaira Faqiri, ${ }^{2}$ Zubair Ahmad ${ }^{0},{ }^{3}$ Walid Emam, ${ }^{4}$ M. Yusuf, ${ }^{5}$ \\ and A. M. Sharawy ${ }^{6}$ \\ ${ }^{1}$ School of Finance, Shanghai Lixin University of Accounting and Finance, Shanghai, China \\ ${ }^{2}$ Education Faculty, Farah Institute of Higher Education, Farah, Afghanistan \\ ${ }^{3}$ Department of Statistics, Yazd University, P.O. Box 89175-741, Yazd, Iran \\ ${ }^{4}$ Department of Statistics and Operations Research College of Science, King Saud University, P. O. Box 2455, \\ Riyadh 11451, Saudi Arabia \\ ${ }^{5}$ Department of Mathematics, Faculty of Science, Helwan University, Helwan, Egypt \\ ${ }^{6}$ Department of Mathematical and Natural Sciences, Faculty of Engineering, Egyptian Russian University, Badr, Egypt
}

Correspondence should be addressed to Zubair Ahmad; z.ferry21@gmail.com

Received 23 March 2021; Revised 7 April 2021; Accepted 19 April 2021; Published 4 May 2021

Academic Editor: Ahmed Mostafa Khalil

Copyright $\left({ }^{2} 2021\right.$ Jin Zhao et al. This is an open access article distributed under the Creative Commons Attribution License, which permits unrestricted use, distribution, and reproduction in any medium, provided the original work is properly cited.

\begin{abstract}
The uses of statistical distributions for modeling real phenomena of nature have received considerable attention in the literature. The recent studies have pointed out the potential of statistical distributions in modeling data in applied sciences, particularly in financial sciences. Among them, the two-parameter Lomax distribution is one of the prominent models that can be used quite effectively for modeling data in management sciences, banking, finance, and actuarial sciences, among others. In the present article, we introduce a new three-parameter extension of the Lomax distribution via using a class of claim distributions. The new model may be called the Lomax-Claim distribution. The parameters of the Lomax-Claim model are estimated using the maximum likelihood estimation method. The behaviors of the maximum likelihood estimators are examined by conducting a brief Monte Carlo study. The potentiality and applicability of the Lomax claim model are illustrated by analyzing a dataset taken from financial sciences representing the vehicle insurance loss data. For this dataset, the proposed model is compared with the Lomax, power Lomax, transmuted Lomax, and exponentiated Lomax distributions. To show the best fit of the competing distributions, we consider certain analytical tools such as the Anderson-Darling test statistic, Cramer-Von Mises test statistic, and Kolmogorov-Smirnov test statistic. Based on these analytical measures, we observed that the new model outperforms the competitive models. Furthermore, a bivariate extension of the proposed model called the Farlie-Gumble-Morgenstern bivariate Lomax-Claim distribution is also introduced, and different shapes for the density function are plotted. An application of the bivariate model to GDP and export of goods and services is provided.
\end{abstract}

\section{Introduction}

The Lomax or Pareto II (the shifted Pareto) distribution was proposed by Lomax in the mid of the last century to model business failure data. This model has a wide range of applications in a variety of fields, particularly, in income and wealth inequality, size of cities, and financial and actuarial sciences. Furthermore, it has been applied to model income and wealth data, the size distribution of computer files on servers, reliability, life testing, and curve analysis [1].
A random variable say $X$ is said to follow the two-parameter Lomax distribution if its cumulative distribution function (cdf) denoted by $F(x ; \xi)$ is given by

$$
F(x ; \xi)=1-(1+\gamma x)^{-\alpha}, \quad x \geq 0, \alpha, \gamma>0,
$$

where $\xi=(\alpha, \gamma)$. The probability density function (pdf), survival function (sf), and hazard rate function (hrf) of the Lomax random variable are given by 


$$
\begin{aligned}
& f(x ; \xi)=\alpha \gamma(1+\gamma x)^{-\alpha-1}, \quad x>0, \\
& S(x ; \xi)=(1+\gamma x)^{-\alpha}, \quad x>0, \\
& h(x ; \xi)=\frac{\alpha \gamma}{(1+\gamma x)}, \quad x>0,
\end{aligned}
$$

respectively.

The Lomax model can be obtained in a number of ways. It can be obtained as a special form of the Pearson type VI distribution. It is also considered a mixture of the exponential and gamma distributions. In the context lifetime scenario, the Lomax distribution falls under the domain of decreasing failure rate distributions. This distribution has been proved as a significant alternative to the exponential, Weibull, and gamma distributions to model heavy-tailed data sets. Due to the importance and applicability of the Lomax distribution, it has been extensively generalized and modified to obtain a more flexible extension of the Lomax distributions, for example, power Lomax distribution [2], transmuted Lomax distribution [3], exponentiated Lomax distribution [4], weighted Lomax distribution [5], exponential Lomax distribution [6], gamma Lomax distribution [7], Poisson Lomax distribution [8], an extended Lomax distribution [9], Marshall-Olkin extended Lomax [10], exponentiated Weibull Lomax [11], Kumaraswamy generalized power Lomax [12], Marshall-Olkin length biased Lomax [13], Gompertz Lomax [14], half-logistic Lomax [15], Gumbel Lomax [16], transmuted Weibull Lomax [17], and transmuted exponentiated Lomax [18].

In this article, we focus on proposing a new three-parameter modification of the Lomax distribution called the Lomax-Claim (L-Claim) distribution for modeling financial data. The L-Claim distribution is introduced by adopting the approach of a class of claim distributions of Ahmad et al. [19]. The cdf and pdf of a class of claim distributions are given, respectively, by

$$
\begin{aligned}
& G(x ; \sigma, \xi)=\frac{\sigma(F(x ; \xi))^{2}}{1-(1-\sigma) F(x ; \xi)}, \quad x \in \mathbb{R}, \\
& g(x ; \sigma, \xi)=\frac{\sigma f(x ; \xi) F(x ; \xi)[2-(1-\sigma) F(x ; \xi)]}{[1-(1-\sigma) F(x ; \xi)]^{2}}, \quad x \in \mathbb{R} .
\end{aligned}
$$

In this article, the L-Claim distribution along with its statistical properties will be given intensive statistical treatment. However, the flexibility and applicability of the LClaim distribution are examined by an application to the insurance loss insurance data.

The rest of this paper is carried out as follows. In Section 2, the L-Claim distribution is introduced. Section 3 is devoted to the bivariate extension of the L-Claim distribution. The quantile function and some possible plots for the mean, variance, skewness, and kurtosis are provided in Section 4. Section 5 is devoted to the maximum likelihood estimation of the L-Claim distribution. The Monte Carlo simulation study is conducted in Section 6. A real-life application of the L-Claim model to insurance loss data Farlie-Gumble-Morgenstern bivariate Lomax-Claim (FGMBL-Claim) is applied to the GDP and export of goods and services is provided in Section 8. Some concluding remarks are given in the last section.

\section{The L-Claim Distribution}

In the following section, we study a three-parameter L-Claim distribution and investigate the shapes of its pdf. The cdf and pdf of the L-Claim distribution can be obtained by substituting expressions (1) and (2) in (3) and (4), respectively.

A random variable $X$ has the L-Claim distribution if its cdf is given by

$G(x ; \sigma, \xi)=\frac{\sigma\left(1-(1+\gamma x)^{-\alpha}\right)^{2}}{1-(1-\sigma)\left(1-(1+\gamma x)^{-\alpha}\right)}, \quad x \geq 0, \alpha, \gamma, \sigma>0$.

The pdf corresponding to (5) is given by

$$
g(x ; \sigma, \xi)=\frac{\alpha \gamma \sigma(1+\gamma x)^{-\alpha-1}\left(1-(1+\gamma x)^{-\alpha}\right)\left[2-(1-\sigma)\left(1-(1+\gamma x)^{-\alpha}\right)\right]}{\left[1-(1-\sigma)\left(1-(1+\gamma x)^{-\alpha}\right)\right]^{2}}, \quad x>0 .
$$

Some possible behaviors of the pdf of the L-Claim distribution are shown in Figure 1. The plots in the right panel of Figure 1 are sketched for $\alpha=0.9, \gamma=1$, and $\sigma=1.2$ (red-line), $\alpha=1.2, \gamma=1$, and $\sigma=3.5$ (green-line), and $\alpha=$ $2.5, \gamma=1$, and $\sigma=4.2$ (black-line), whereas the plots in the left panel of Figure 1 are presented for $\alpha=1.8, \gamma=1$, and $\sigma=1.6$ (red-line), $\alpha=1.4, \gamma=1$, and $\sigma=$ 1.8 (green-line), and $\alpha=3.5, \gamma=1$, and $\sigma=2.5$ (black-line).
From Figure 1, it is clear that when the parameters $\alpha$ and $\sigma$ increase and $\gamma<1$, then the proposed L-Claim posses heavy tails. Due to the right-skewed and heavy-tailed behavior of the proposed model, it can be a good candidate model for modeling heavy-tailed data which are very important in financial and actuarial sciences.

The sf and hrf of the L-Claim distributed random variable are given by 


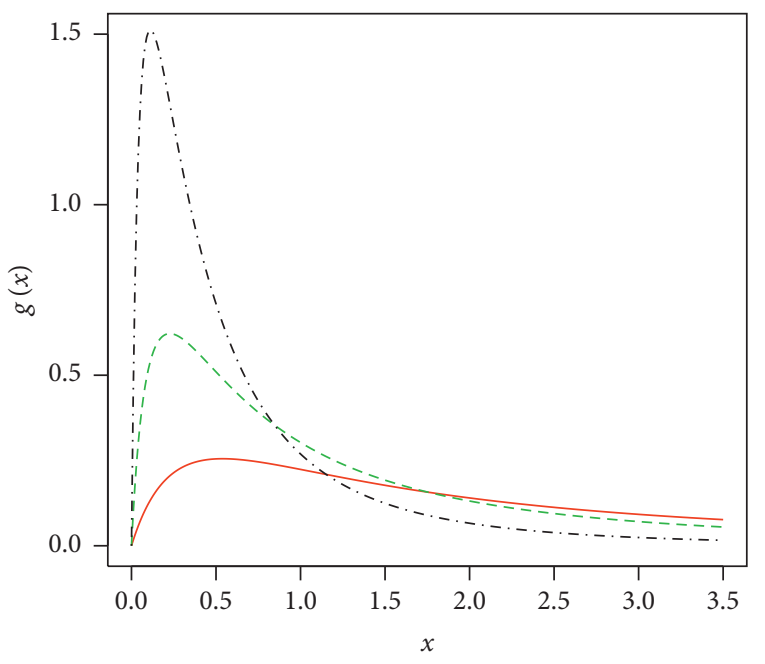

(a)

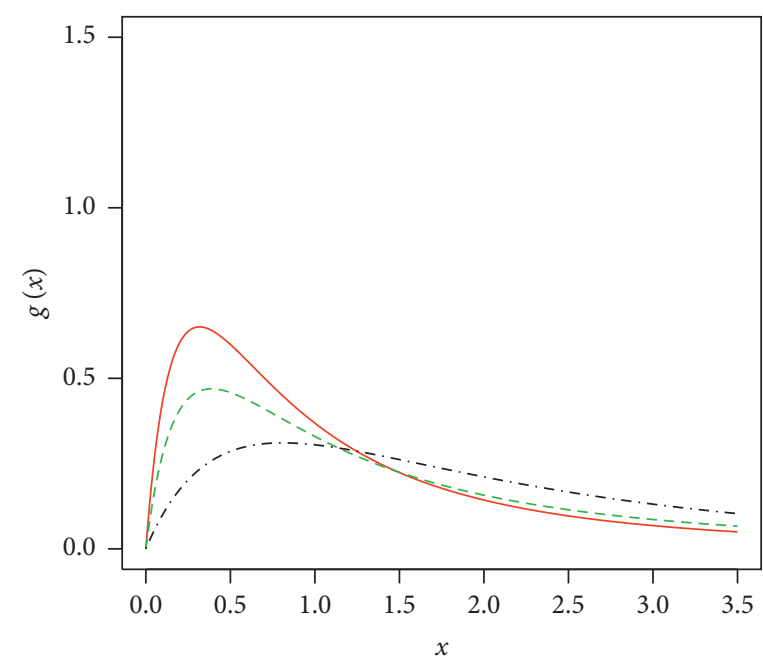

(b)

Figure 1: Plots for the pdf of the L-Claim distribution for selected parameter values.

$$
\begin{aligned}
& S(x ; \sigma, \boldsymbol{\xi})=\frac{1-(1-\sigma)\left(1-(1+\gamma x)^{-\alpha}\right)-\sigma\left(1-(1+\gamma x)^{-\alpha}\right)^{2}}{1-(1-\sigma)\left(1-(1+\gamma x)^{-\alpha}\right)}, \quad x>0, \\
& h(x ; \sigma, \xi)=\frac{\alpha \gamma \sigma(1+\gamma x)^{-\alpha-1}\left(1-(1+\gamma x)^{-\alpha}\right)\left[2-(1-\sigma)\left(1-(1+\gamma x)^{-\alpha}\right)\right]}{\left(1-(1-\sigma)\left(1-(1+\gamma x)^{-\alpha}\right)-\sigma\left(1-(1+\gamma x)^{-\alpha}\right)^{2}\right)} \times \frac{1}{\left[1-(1-\sigma)\left(1-(1+\gamma x)^{-\alpha}\right)\right]}, \quad x>0,
\end{aligned}
$$

respectively. Some possible behaviors of the hrf of the L-Claim distribution are shown in Figure 2. The plots in the right panel of Figure 2 are sketched for $\alpha=0.9, \gamma=1$, and $\sigma=1.2$ (redline), $\alpha=1.2, \gamma=1$, and $\sigma=3.5$ (green-line), and $\alpha=2.5, \gamma=$ 1 , and $\sigma=4.2$ (black-line), whereas the plots in the left panel of Figure 2 are presented for $\alpha=4.8, \gamma=0.3$, and $\sigma=1.1$ (redline), $\alpha=0.9, \gamma=1$, and $\sigma=1.8$ (green-line), and $\alpha=3.2, \gamma=$ 1 , and $\sigma=1.2$ (black-line).

As we stated above, the two-parameter traditional Lomax distribution belongs to the class of decreasing failure rate distributions. However, from the plots provided in Figure 2, it is clear that the L-Claim distribution has unimodal and increasing failure rate functions. Henceforth, besides the heavy-tailed behavior, this is another superiority of the proposed model over the Lomax distribution.

\section{Farlie-Gumble-Morgenstern Bivariate Lomax-Claim Distribution}

Copula functions are used to represent the joint cdf of the two marginal univariate distributions. If $G\left(x_{j}\right)$ is the univariate cdf of $X_{j}=j=1,2$, the joint cdf denoted by $G\left(x_{1}, x_{2}\right)$ is defined by the copula function given by

$$
G\left(x_{1}, x_{2}\right)=C_{\rho}\left(G\left(x_{1}\right), G\left(x_{2}\right)\right),
$$

where $\rho$ is the dependence measures between $X_{1}$ and $X_{2}$. The joint cdf and pdf of the Farlie-Gumble-Morgenstern (FGM) copula (Conway [20]) are given, respectively, by

$$
\begin{aligned}
G\left(x_{1}, x_{2}\right)= & G\left(x_{1}\right) G\left(x_{2}\right)\left[1+\rho\left(1-G\left(x_{1}\right)\right)\left(1-G\left(x_{2}\right)\right)\right], \\
& -1<\rho<1, \\
g\left(x_{1}, x_{2}\right)= & g\left(x_{1}\right) g\left(x_{2}\right)\left[1+\rho\left(1-2 G\left(x_{1}\right)\right)\left(1-2 G\left(x_{2}\right)\right)\right] .
\end{aligned}
$$

The random variables say $\left(X_{1}, X_{2}\right)$ follow the FGMBLClaim distribution if its cdf is defined by

$$
\begin{aligned}
G\left(x_{1}, x_{2}\right)= & \frac{\sigma_{1}\left(1-\left(1+\gamma_{1} x\right)^{-\alpha_{1}}\right)^{2}}{\left[1-\left(1-\sigma_{1}\right)\left(1-\left(1+\gamma_{1} x\right)^{-\alpha_{1}}\right)\right]} \frac{\sigma_{2}\left(1-\left(1+\gamma_{2} x\right)^{-\alpha_{2}}\right)^{2}}{\left[1-\left(1-\sigma_{2}\right)\left(1-\left(1+\gamma_{2} x\right)^{-\alpha_{2}}\right)\right]} \\
& \times\left[1+\rho\left(1-\frac{\sigma_{1}\left(1-\left(1+\gamma_{1} x\right)^{-\alpha_{1}}\right)^{2}}{1-\left(1-\sigma_{1}\right)\left(1-\left(1+\gamma_{1} x\right)^{-\alpha_{1}}\right)}\right)\left(1-\frac{\sigma_{2}\left(1-\left(1+\gamma_{2} x\right)^{-\alpha_{2}}\right)^{2}}{1-\left(1-\sigma_{2}\right)\left(1-\left(1+\gamma_{2} x\right)^{-\alpha_{2}}\right)}\right)\right],
\end{aligned}
$$




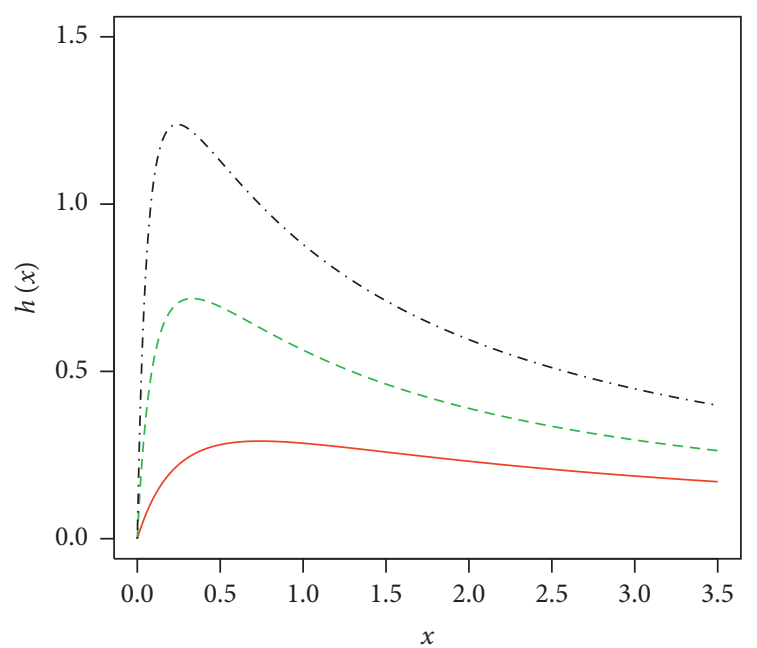

(a)

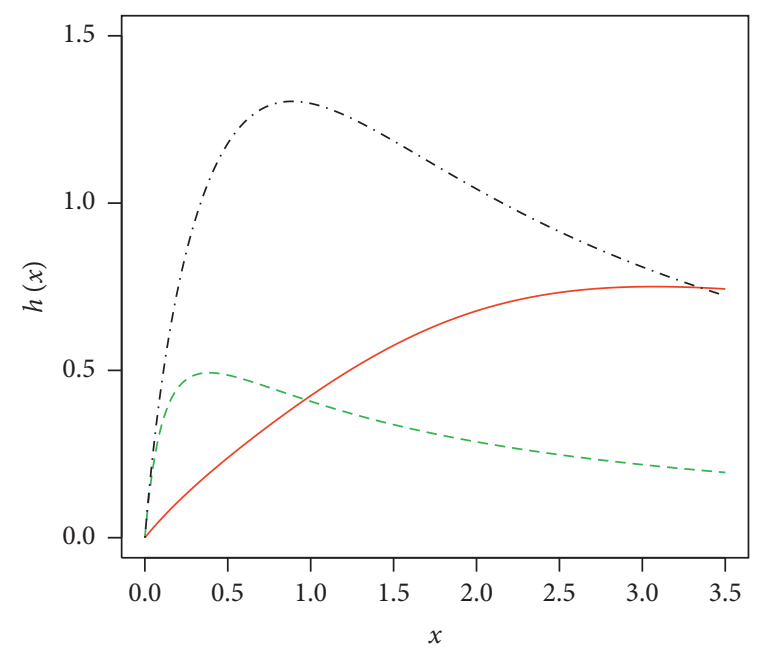

(b)

Figure 2: Plots for the hrf of the L-Claim distribution for selected parameter values.

where $x_{1}, x_{2}, \alpha_{1}, \gamma_{1}, \sigma_{1}, \alpha_{2}, \gamma_{2}, \sigma_{2}>0$. The corresponding pdf

is

$$
\begin{aligned}
& g\left(x_{1}, x_{2}\right)= \frac{\alpha_{1} \gamma_{1} \sigma_{1}\left(1+\gamma_{1} x\right)^{-\alpha_{1}-1}\left(1-\left(1+\gamma_{1} x\right)^{-\alpha_{1}}\right)\left[2-\left(1-\sigma_{1}\right)\left(1-\left(1+\gamma_{1} x\right)^{-\alpha_{1}}\right)\right]}{\left[1-\left(1-\sigma_{1}\right)\left(1-\left(1+\gamma_{1} x\right)^{-\alpha_{1}}\right)\right]^{2}} \\
& \times \frac{\alpha_{2} \gamma_{2} \sigma_{2}\left(1+\gamma_{2} x\right)^{-\alpha_{2}-1}\left(1-\left(1+\gamma_{2} x\right)^{-\alpha_{2}}\right)\left[2-\left(1-\sigma_{2}\right)\left(1-\left(1+\gamma_{2} x\right)^{-\alpha_{2}}\right)\right]}{\left[1-\left(1-\sigma_{2}\right)\left(1-\left(1+\gamma_{2} x\right)^{-\alpha_{2}}\right)\right]^{2}} \\
& \times\left[1+\rho\left(1-\frac{2 \sigma_{1}\left(1-\left(1+\gamma_{1} x\right)^{-\alpha_{1}}\right)^{2}}{1-\left(1-\sigma_{1}\right)\left(1-\left(1+\gamma_{1} x\right)^{-\alpha_{1}}\right)}\right)\left(1-\frac{2 \sigma_{2}\left(1-\left(1+\gamma_{2} x\right)^{-\alpha_{2}}\right)^{2}}{1-\left(1-\sigma_{2}\right)\left(1-\left(1+\gamma_{2} x\right)^{-\alpha_{2}}\right)}\right)\right] . \\
& \text { pkewness }=\frac{Q(1 / 4)+Q(3 / 4)-2 Q(1 / 2)}{Q(3 / 4)-Q(1 / 4)},
\end{aligned}
$$

provided in Figures 3 and 4 . Whereas plots for the cdf and sf of the FGMBL-Claim distribution are provided in Figure 5.

\section{Quantile Function}

The quantile function of distribution is very useful to apply for generating random numbers by Monte Carlo simulation. Suppose $X$ follows the L-Claim distribution, then the quantile function of $X$ can be obtained via inverting $G(x ; \sigma, \xi)=u$ in (5). We obtain

$$
x_{q}=G^{-1}(u), \quad u \in(0,1) .
$$

The nonlinear expression provided in (12) can be used to obtain the random numbers from the L-Claim distribution.

Furthermore, the effects of the shape parameters on the skewness and kurtosis can be detected on quantile measures. We obtain skewness and kurtosis measures of the L-Claim distribution using (12). Bowley's skewness (Bowley [21]) of $X$ is given as follows: whereas Moor's kurtosis (Moor [22]) is as follows:

$$
\text { kurtosis }=\frac{Q(7 / 8)-Q(5 / 8)+Q(3 / 8)-Q(1 / 8)}{Q(6 / 8)-Q(2 / 8)} .
$$

These measures are less sensitive to outliers. Moreover, they do exist for distributions without moments. Some possible plots for the mean, variance, skewness, and kurtosis of the L-Claim distribution are provided in Figures 6 and 7 .

\section{Parameters Estimation}

Several approaches for the estimation of the unknown parameters have been studied and applied in the literature. Among them, the method of maximum likelihood estimation is the most widely applied approach. The estimators obtained via this method possess several desirable properties and can be used quite effectively for constructing confidence bounds. In this section, we adopt this method to obtain the estimators of the model parameters. Let $x_{1}, x_{2}, \ldots, x_{n}$ be 
observed values of the random sample taken from the L-Claim distribution with parameters $\alpha, \gamma$, and $\sigma$. The $\log$ likelihood function corresponding to (9) is given by

$$
\begin{aligned}
\ell= & n \log \alpha+n \log \gamma+n \log \sigma-(\alpha-1) \sum_{i=1}^{n} \log \left(1+\gamma x_{i}\right) \\
& +\sum_{i=1}^{n} \log \left(1-\left(1+\gamma x_{i}\right)^{-\alpha}\right) \\
& +\sum_{i=1}^{n} \log \left[2-(1-\sigma)\left(1-\left(1+\gamma x_{i}\right)^{-\alpha}\right)\right] \\
& -2 \sum_{i=1}^{n} \log \left[1-(1-\sigma)\left(1-\left(1+\gamma x_{i}\right)^{-\alpha}\right)\right] .
\end{aligned}
$$

The expression provided in (8) can be characteristically solved by using Newton's approach or by fixed-point iteration methods. The partial derivatives of (8), on behalf of parameters, are

$$
\begin{aligned}
& \frac{\partial \ell}{\partial \alpha}=\frac{n}{\alpha}-\sum_{i=1}^{n} \log \left(1+\gamma x_{i}\right)+\sum_{i=1}^{n} \frac{\log \left(1+\gamma x_{i}\right)\left[\left(1+\gamma x_{i}\right)^{-\alpha}\right]}{\left(1-\left(1+\gamma x_{i}\right)^{-\alpha}\right)} \\
& +2(1-\sigma) \sum_{i=1}^{n} \frac{\log \left(1+\gamma x_{i}\right)\left[\left(1+\gamma x_{i}\right)^{-\alpha}\right]}{\left[1-(1-\sigma)\left(1-\left(1+\gamma x_{i}\right)^{-\alpha}\right)\right]} \\
& -(1-\sigma) \sum_{i=1}^{n} \frac{\log \left(1+\gamma x_{i}\right)\left[\left(1+\gamma x_{i}\right)^{-\alpha}\right]}{\left[2-(1-\sigma)\left(1-\left(1+\gamma x_{i}\right)^{-\alpha}\right)\right]} \\
& \frac{\partial \ell}{\partial \gamma}=\frac{n}{\gamma}-(\alpha-1) \sum_{i=1}^{n} \frac{x_{i}}{\left(1+\gamma x_{i}\right)}+\alpha \sum_{i=1}^{n} \frac{x_{i}\left(1+\gamma x_{i}\right)^{-\alpha-1}}{\left(1-\left(1+\gamma x_{i}\right)^{-\alpha}\right)} \\
& -\alpha(1-\sigma) \sum_{i=1}^{n} \frac{x_{i}\left(1+\gamma x_{i}\right)^{-\alpha-1}}{\left[2-(1-\sigma)\left(1-\left(1+\gamma x_{i}\right)^{-\alpha}\right)\right]} \\
& +(1-\sigma) \sum_{i=1}^{n} \frac{x_{i}\left(1+\gamma x_{i}\right)^{-\alpha-1}}{\left[1-(1-\sigma)\left(1-\left(1+\gamma x_{i}\right)^{-\alpha}\right)\right]}, \\
& \frac{\partial \ell}{\partial \sigma}=\frac{n}{\sigma}+\sum_{i=1}^{n} \frac{\left(1-\left(1+\gamma x_{i}\right)^{-\alpha}\right)}{\left[2-(1-\sigma)\left(1-\left(1+\gamma x_{i}\right)^{-\alpha}\right)\right]} \\
& -2 \sum_{i=1}^{n} \frac{\left(1-\left(1+\gamma x_{i}\right)^{-\alpha}\right)}{\left[1-(1-\sigma)\left(1-\left(1+\gamma x_{i}\right)^{-\alpha}\right)\right]} .
\end{aligned}
$$

\section{Monte Carlo Simulation Study}

This section deals with assessing the performance of the maximum likelihood estimators of the L-Claim distribution by the Monte Carlo simulation study. The simulation is performed for two different sets of parameters of the LClaim distribution. The simulation study is carried out as follows:
(1) Random samples of different sizes $n=25,50, \ldots, 750$ are generated from L-Claim distribution.

(2) The model parameters have been estimated via the maximum likelihood method.

(3) 750 repetitions are made to calculate the biases, absolute biases, and mean square errors (MSEs) of these estimators.

(4) The formulas for obtaining the biases and MSEs are given by

$$
\begin{aligned}
& \operatorname{Bias}(\widehat{\alpha})=\frac{1}{750} \sum_{i=1}^{750}\left(\widehat{\alpha}_{i}-\alpha\right), \\
& \operatorname{MSE}(\widehat{\alpha})=\frac{1}{750} \sum_{i=1}^{750}\left(\widehat{\alpha}_{i}-\alpha\right)^{2},
\end{aligned}
$$

respectively.

(5) Step (4), is also repeated for the parameters $(\gamma, \sigma)$.

For the simulated dataset 1, the box plot and Kernel density estimator are presented in Figure 8 . Whereas for the simulated dataset 2, the box plot and Kernel density estimator are presented in Figure 9.

The simulation results of the L-Claim distribution are presented in Tables 1 and 2 . To support the results provided in Tables 1 and 2, the simulation results are also displayed graphically in Figures 10 and 11 .

\section{Application of the L-Claim to Vehicle Insurance Loss Data}

The main applications of the heavy-tailed distributions are the extreme value theory or insurance loss phenomena. In this section, we illustrate the applicability of the L-Claim distribution by analyzing the vehicle insurance loss data. The data are available at http://www. businessandeconomics.mq.edu.au. We compare the goodness-of-fit results of the L-Claim distribution with the two-parameter Lomax distribution and three-parameter power Lomax (P-Lomax) distribution [2], transmuted Lomax (T-Lomax) distribution [3], and exponentiated Lomax (E-Lomax) distribution [4]. We estimate the unknown parameters of the fitted distributions via the maximum likelihood method using the Rscript adequacy model with the "Nelder-Mead" method; see Appendix. The goodness-of-fit statistics including the Anderson-Darling (AD), Cramer-Von Mises (CM), and Kolmogorov-Smirnov (KS) with the corresponding $p$ value are used to compare the fitted models.

Table 3 gives the MLEs of the model parameters. Whereas the analytical measures are provided in Table 4. In general, the smaller the values of these measures, the better the fit to the data. After carrying out the analysis, we observe that the L-Claim distribution gives the lowest values for the analytical measures among all the fitted models. So, the L-Claim distribution could be chosen as 

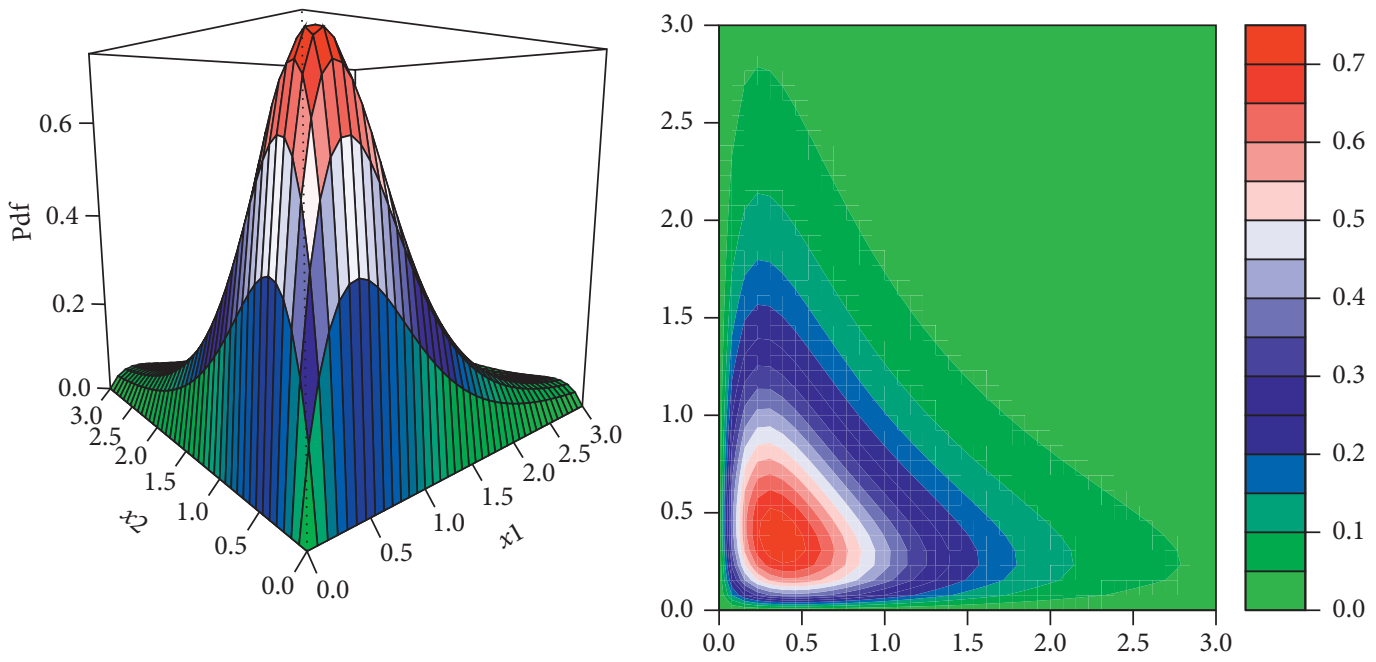

(a)

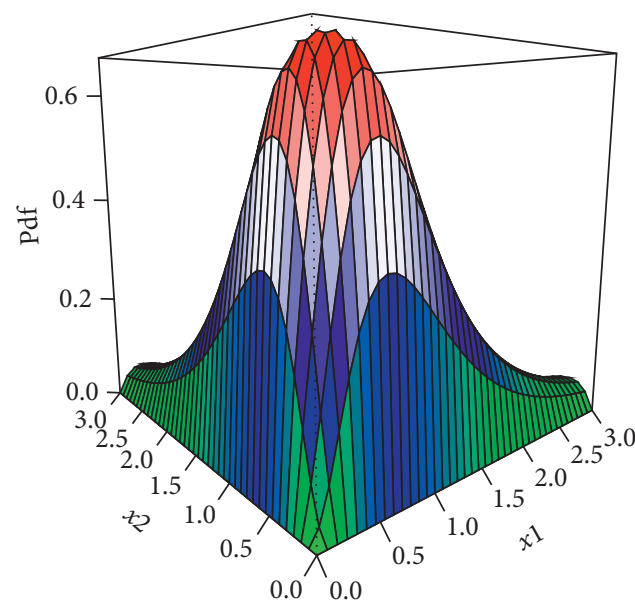

(b)

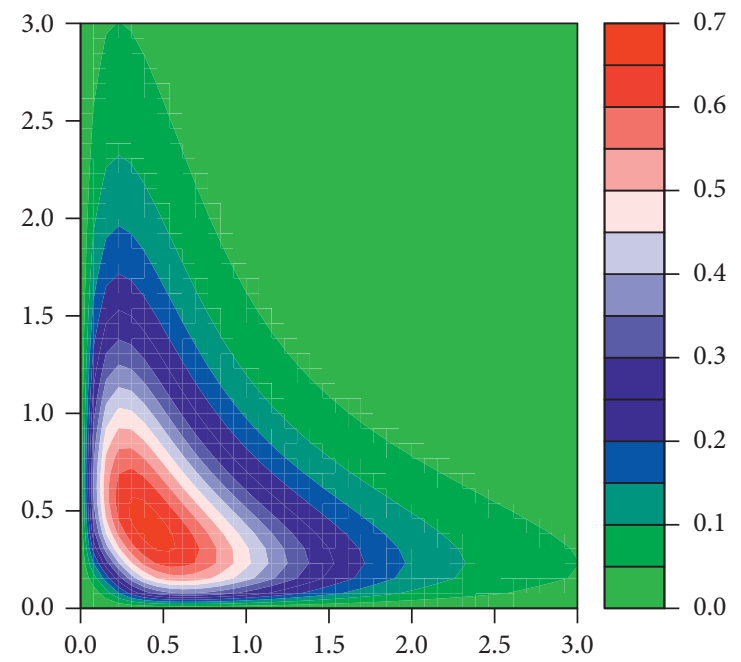

(d)

FIGURE 3: Different plots for the pdf of the FGMBL-Claim distribution for $\alpha_{1}=\alpha_{2}=3, \gamma_{1}=\gamma_{2}=1, \sigma_{1}=\sigma_{2}=0.5$, and $\rho=-0.5$ and $\rho=-1$.
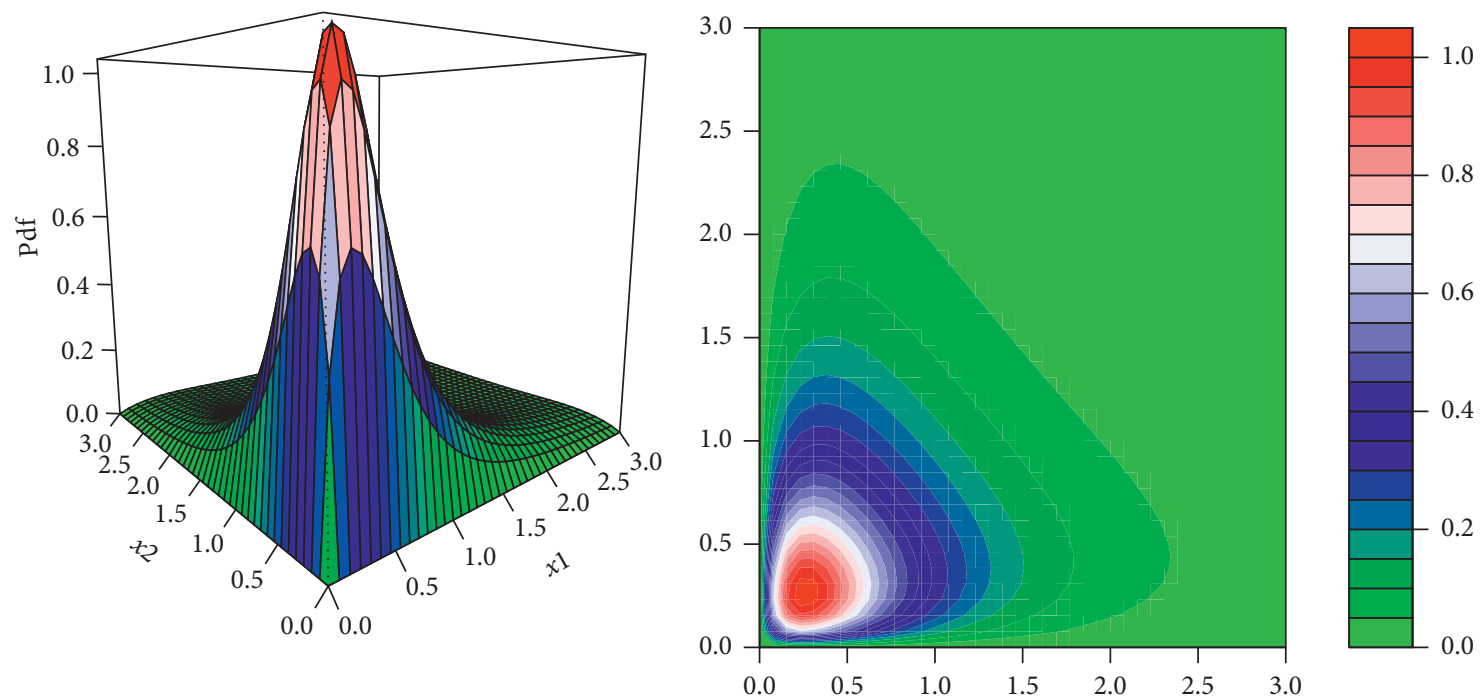

(a)

(b)

FIgURE 4: Continued. 


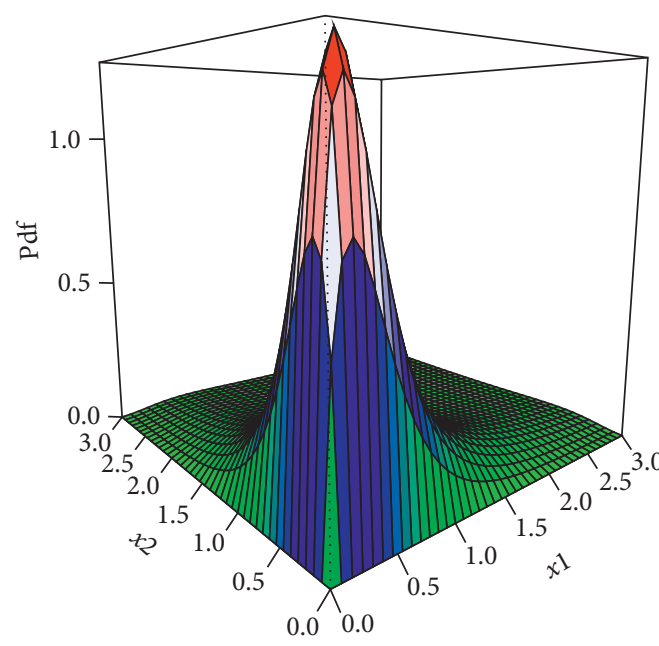

(c)

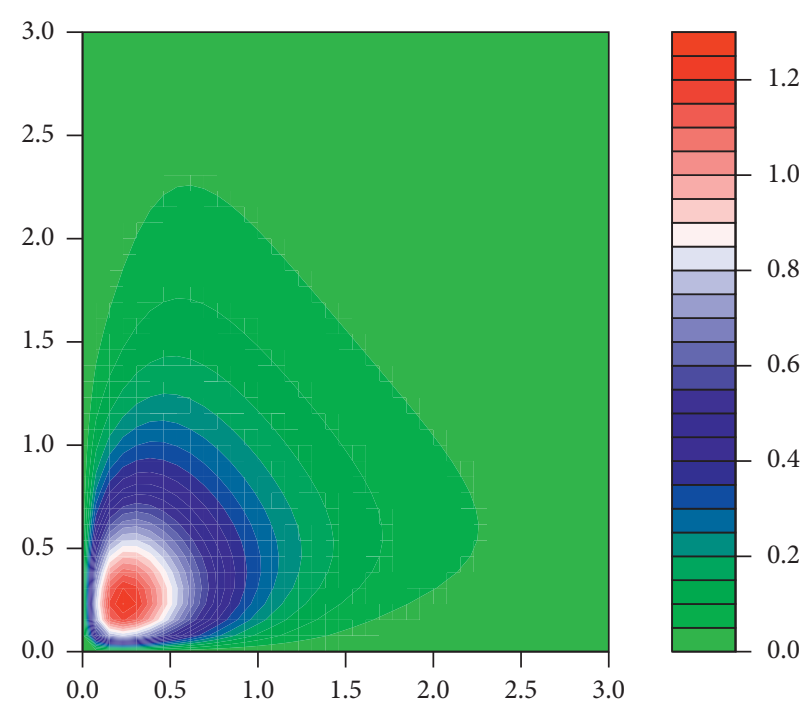

(d)

FIGURE 4: Different plots for the pdf of the FGMBL-Claim distribution for $\alpha_{1}=\alpha_{2}=3, \gamma_{1}=\gamma_{2}=1, \sigma_{1}=\sigma_{2}=0.5$, and $\rho=0.5$ and $\rho=1$.

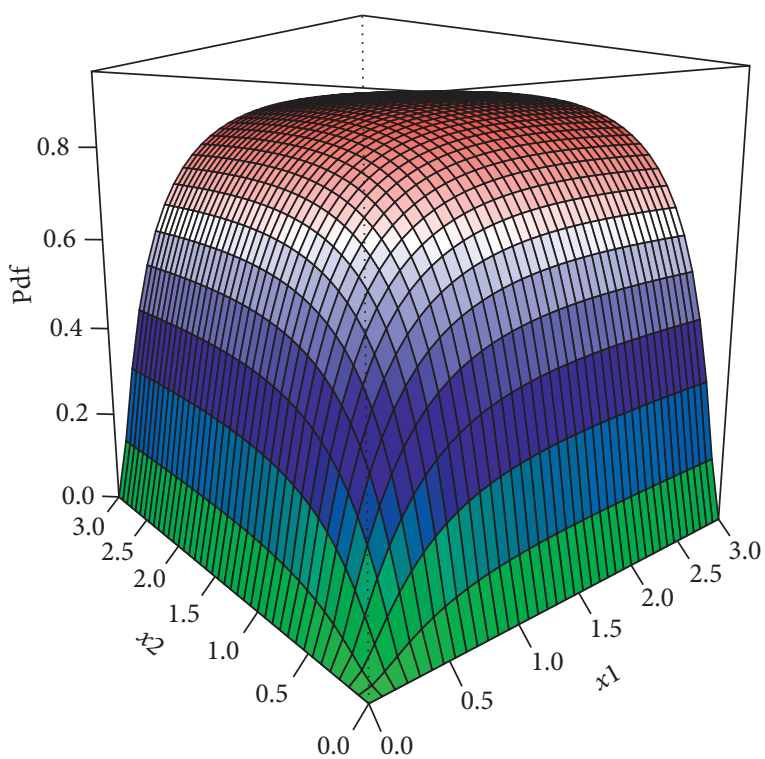

(a)

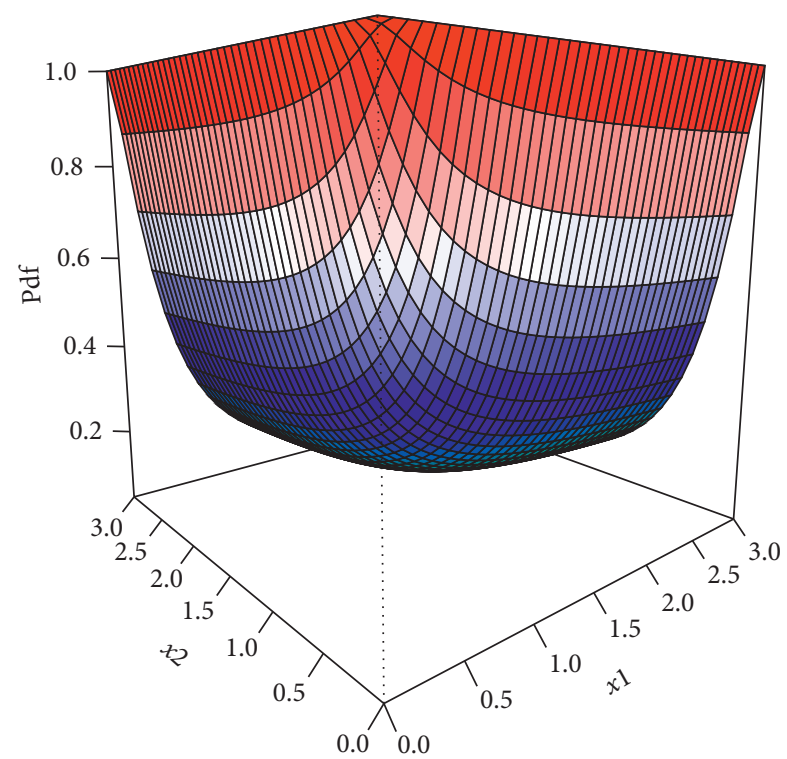

(b)

FIgure 5: Plots for the cdf and sf of the FGMBL-Claim for $\alpha_{1}=\alpha_{2}=3, \gamma_{1}=\gamma_{2}=1, \sigma_{1}=\sigma_{2}=0.5$, and $\rho=0.5$.

the best model for modeling heavy-tailed financial datasets. Furthermore, in support of the results provided in Table 3, the estimated pdf and cdf, Kaplan-Meier survival, and probability-probability (PP) and quantilequantile (QQ) plots of the proposed model along with the box plot of the data are sketched in Figure 12. The plots provided in Figure 12 show that the L-Claim distribution provides a close fit to the insurance loss data.

\section{Application of the FGMBL-Claim to Economics Data}

The economy is an important sector in many developed and developing countries. Therefore, the government and other responsible institutions are always interested in GDP growth and exports of goods and services. To demonstrate the effectiveness of the proposed FGMBL-Claim 


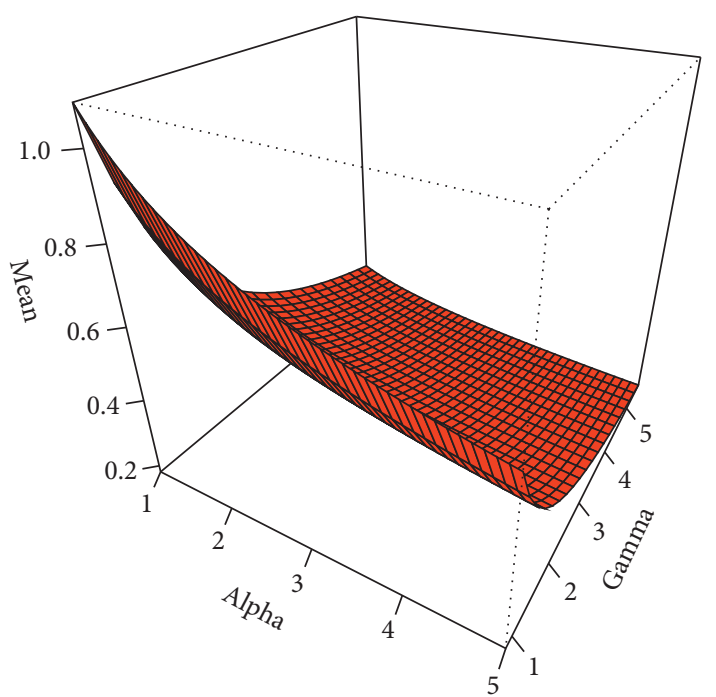

(a)

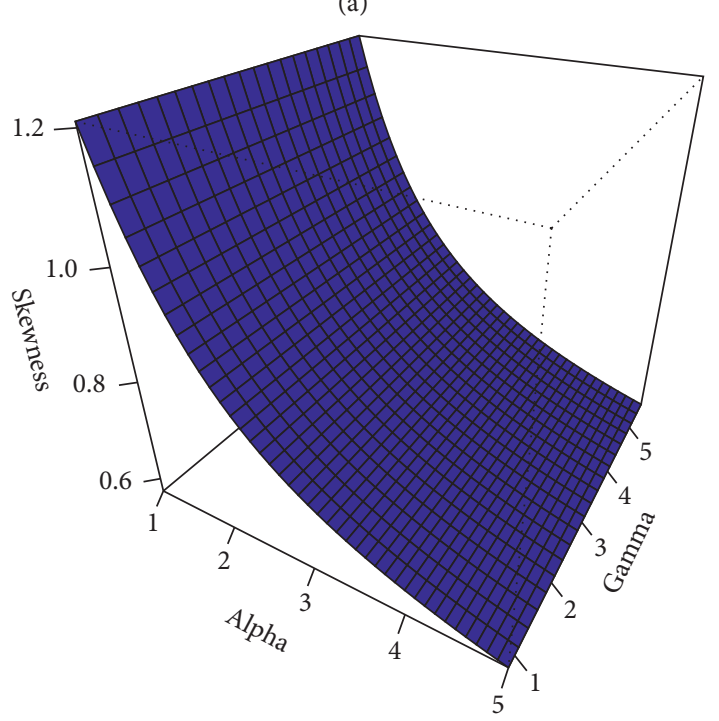

(c)

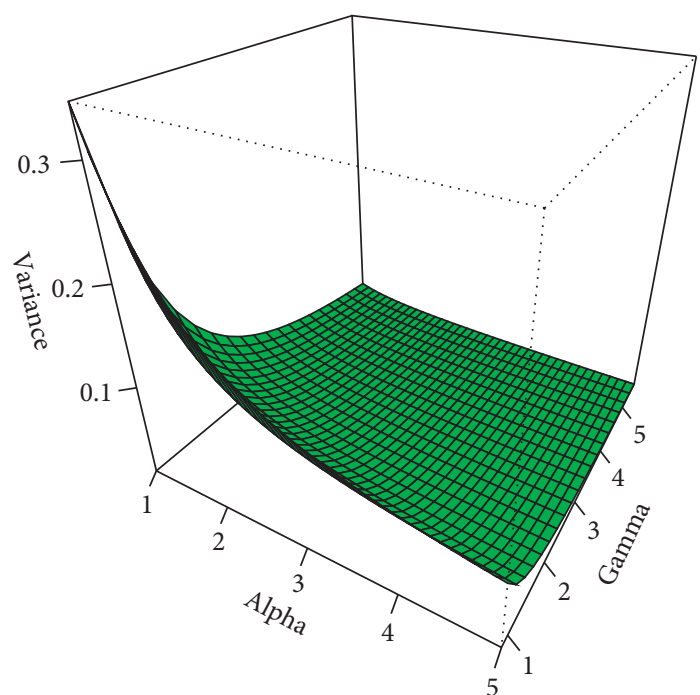

(b)

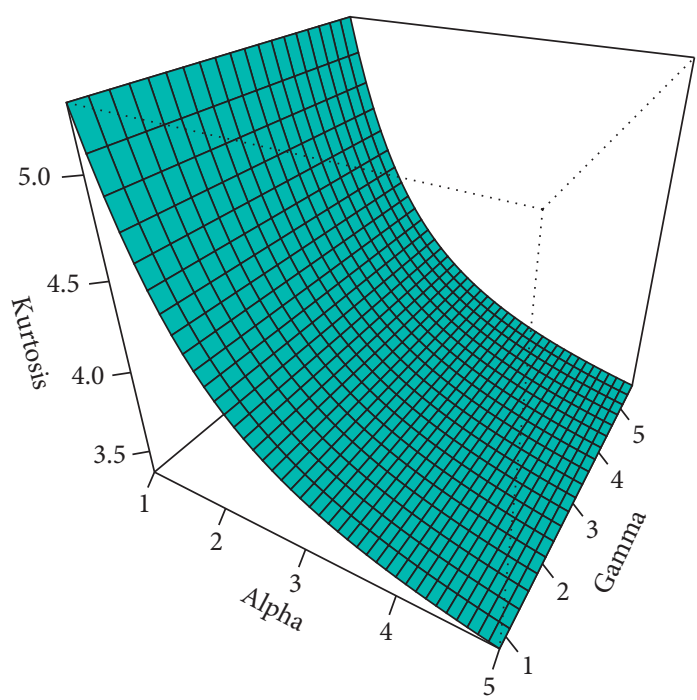

(d)

Figure 6: Plots for the mean, variance, skewness, and kurtosis of the L-Claim distribution for $\gamma=1$ and different values of $\alpha$ and $\sigma$.

distribution, we consider the GDP growth and exports of goods and services. The summary measures of the considered data based on the response variable such as exports of goods and services $\left(X_{1}\right)$ and GDP growth $\left(X_{2}\right)$ are provided in Table 5.

8.1. Testing of Normality of the Economics Data. An assessment of the normality of data is a requirement for many statistical tests because normal data are a basic assumption in parametric testing. There are two main methods of assessing normality such as numerically and graphically. In this section, we check the normality of both the variables $\left(X_{1}, X_{1}\right)$ using the Shapiro-Wilk (SW) normality test and the Anderson-Darling (AD) normality test. Additionally, we use the graphical approach based on the quantile-quantile (QQ) plot. The QQ plot draws the correlation between a given sample and the normal distribution and provides a visual judgment about whether the distribution is bellshaped. It plots a 45-degree reference line and assumes normality if all the points fall approximately along the reference line.

8.1.1. The Shapiro-Wilk Normality Test. The SW normality test can be performed as follows: $H_{0}$ : the data are normally distributed vs. $H_{1}$ : the data are not normally distributed. After applying the SW normality test, we observe that the SW values for $X_{1}$ and $X_{2}$ are, respectively, given by 0.9421 and 0.9336 with corresponding $p$ 


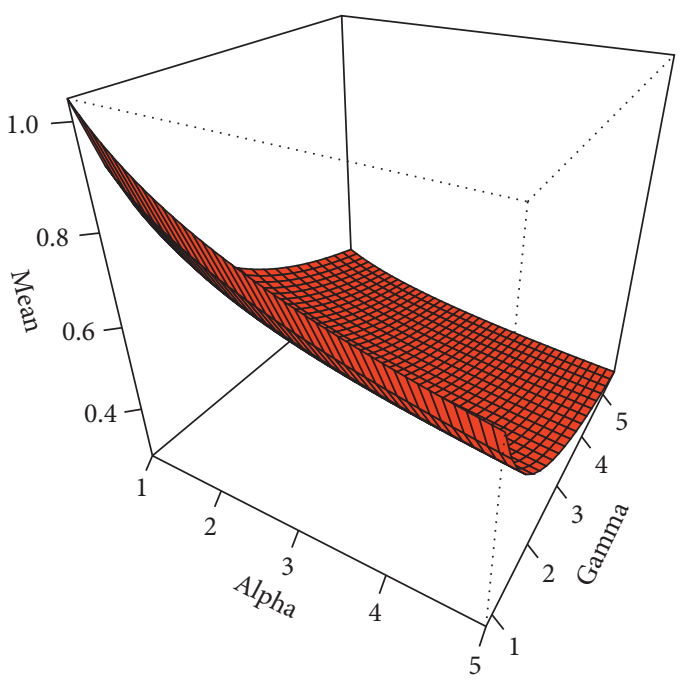

(a)

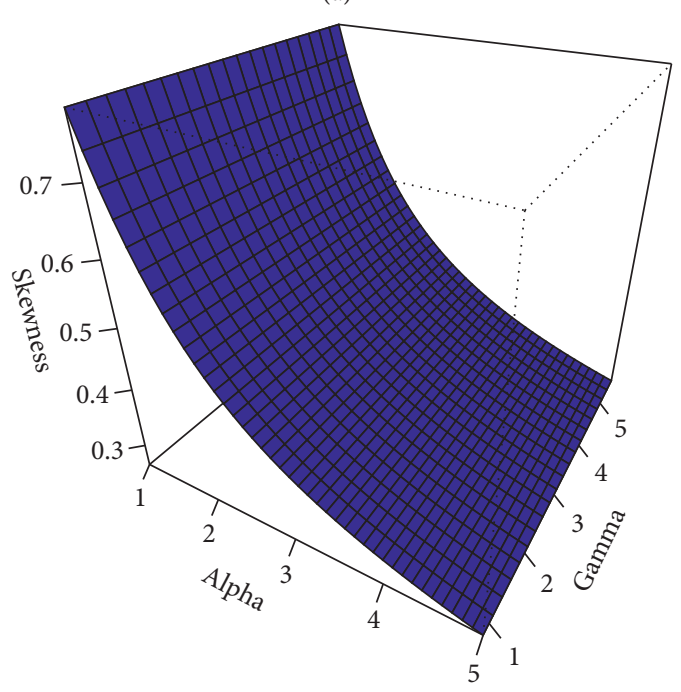

(c)

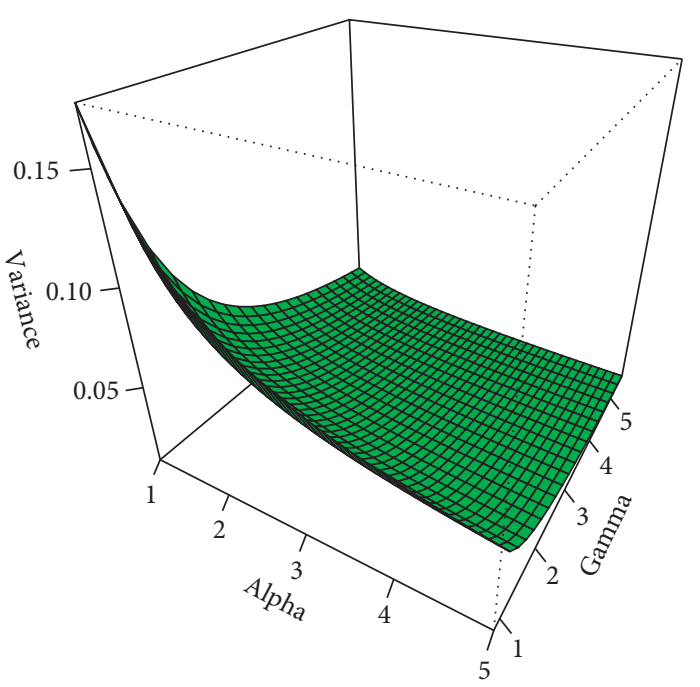

(b)

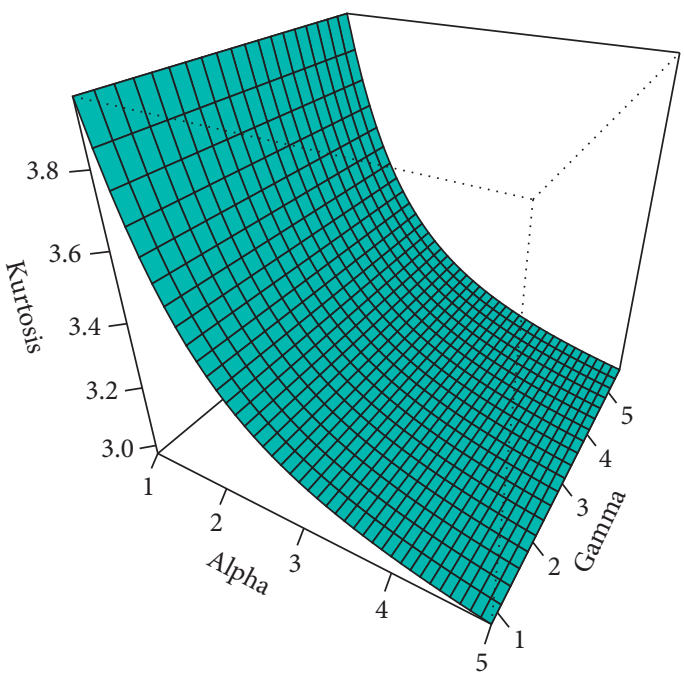

(d)

Figure 7: Plots for the mean, variance, skewness, and kurtosis of the L-Claim distribution for $\gamma=0.5$ and different values of $\alpha$ and $\sigma$.

values give by 0.0948 and 0.0650 , respectively, since the $p$ values are greater than the level of significance 0.05 . Therefore, we conclude that the distributions of the data are significant from the normal distribution. In simple words, we can assume normality. From Figure 13, we can see that the data are normally distributed as all the points are scattered around the diagonal line.

8.1.2. The Anderson-Darling Normality Test. The Anderson-Darling $(\mathrm{AD})$ test is another prominent approach to check the normality of the data. In this subsection, we perform the $\mathrm{AD}$ normality test to check the normality of the data. Under this test, the null hypothesis and alternative hypothesis can be defined as follows: $H_{0}$ : the data are normally distributed vs. $H_{1}$ : the data are not normally distributed. After carrying out the analyses, the values of the $\mathrm{AD}$ normality test statistic for $X_{1}$ and $X_{2}$ are given by 0.6533 and 0.7338 with respective $p$ values give by 0.0798 and 0.0719 . We can see the $p$ values of the AD test statistic is greater than 0.05 . Therefore, we fail to reject the null hypothesis and conclude that the data are normally distributed. From the plots provided in Figure 14, we can see that the data are normally distributed as all the points are scattered around the diagonal line.

8.2. Modeling of the Economics Data. The prime interest of the proposed FMGBL-Claim distribution is to be applied for data analysis purposes, making it useful in many applied fields. Here, this aspect is illustrated by considering an economics dataset based on the GDP growth and exports of goods and services. The total time on test (TTT) plot is an 


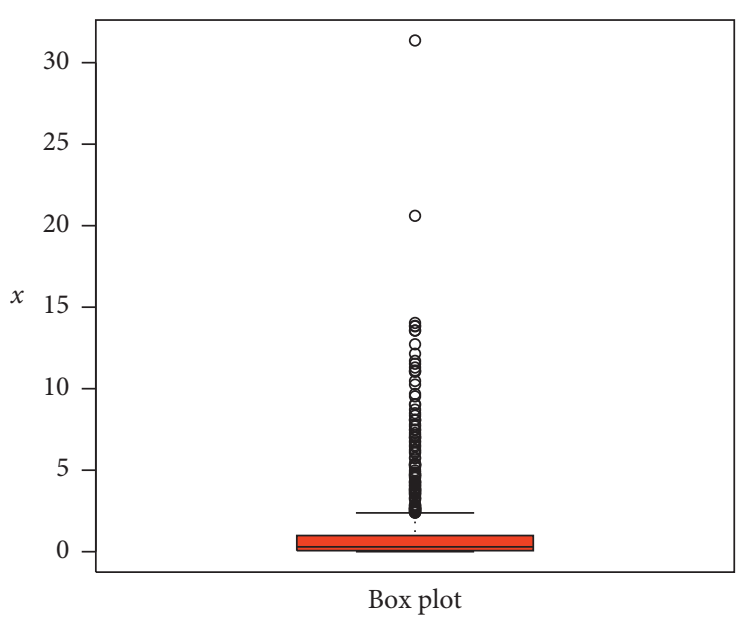

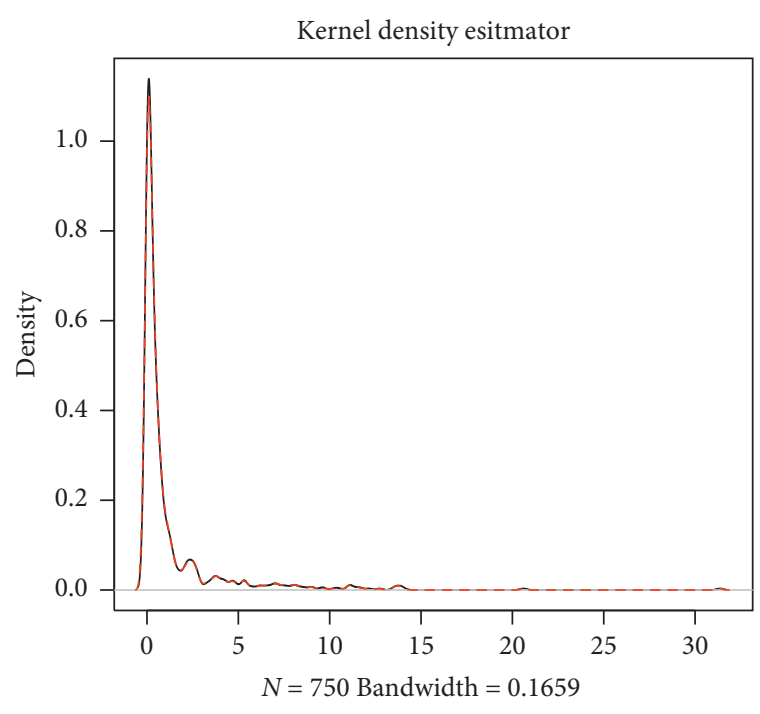

- Simulated dataset 1

- Fitted density

FIgURe 8: Box plot and Kernel density estimator plot of the simulated dataset 1.

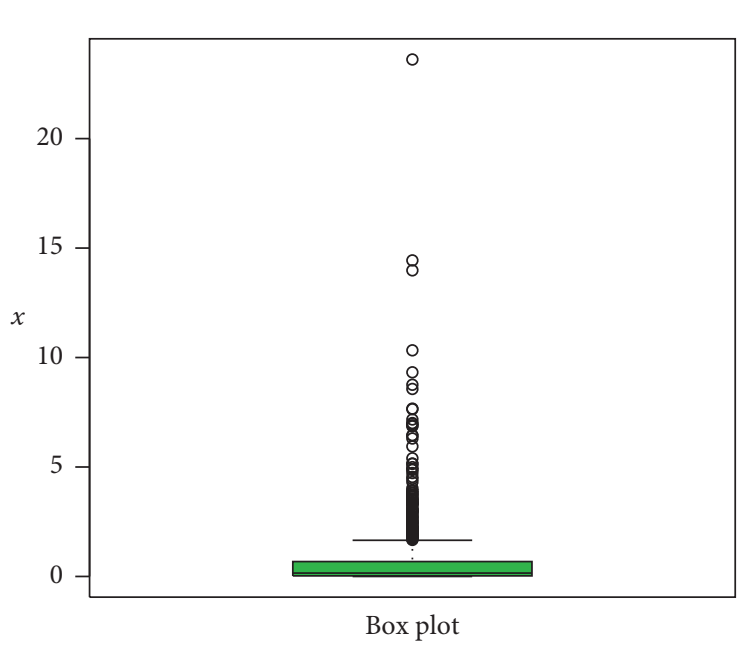

(a)

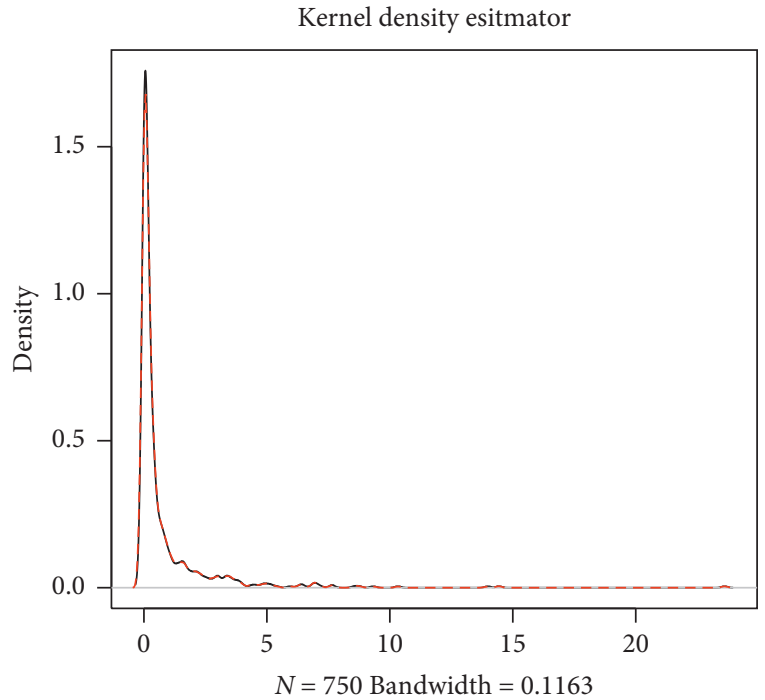

- Simulated dataset 2

- Fitted density

FIgURE 9: Box plot and Kernel density estimator plot of the simulated dataset 2.

empirical plot and used for model identification purposes. The TTT plots of the economics dataset are presented in Figure 15.
In this subsection, we use the economics data to illustrate the proposed model. We prove that the fit power of the FGMBL-Claim model is better than the 
TABLe 1: Simulation results of the L-Claim distribution for $\alpha=0.7, \gamma=0.5$, and $\sigma=1.3$.

\begin{tabular}{|c|c|c|c|c|}
\hline$n$ & Parameters & MLE & Biases & MSEs \\
\hline \multirow{3}{*}{25} & $\widehat{\alpha}$ & 0.740063 & 0.040063 & 0.023912 \\
\hline & $\widehat{\sigma}$ & 1.975112 & 0.675111 & 2.348829 \\
\hline & $\hat{\gamma}$ & 0.646899 & 0.146899 & 0.218631 \\
\hline \multirow{3}{*}{100} & $\widehat{\alpha}$ & 0.708882 & 0.008882 & 0.007295 \\
\hline & $\widehat{\sigma}$ & 1.645087 & 0.345087 & 0.953070 \\
\hline & $\widehat{\gamma}$ & 0.561791 & 0.061791 & 0.077792 \\
\hline \multirow{3}{*}{200} & $\widehat{\alpha}$ & 0.708633 & 0.008633 & 0.003408 \\
\hline & $\widehat{\sigma}$ & 1.411091 & 0.111091 & 0.280088 \\
\hline & $\widehat{\gamma}$ & 0.508988 & 0.008988 & 0.034374 \\
\hline \multirow{3}{*}{400} & $\widehat{\alpha}$ & 0.705445 & 0.005445 & 0.001236 \\
\hline & $\widehat{\sigma}$ & 1.325511 & 0.025511 & 0.044632 \\
\hline & $\hat{\gamma}$ & 0.498914 & 0.007085 & 0.013414 \\
\hline \multirow{3}{*}{600} & $\widehat{\alpha}$ & 0.705628 & 0.005628 & 0.000693 \\
\hline & $\widehat{\sigma}$ & 1.301424 & 0.001424 & 0.011400 \\
\hline & $\widehat{\gamma}$ & 0.490299 & 0.004700 & 0.006831 \\
\hline \multirow{3}{*}{750} & $\widehat{\alpha}$ & 0.705378 & 0.005378 & 0.000528 \\
\hline & $\widehat{\sigma}$ & 1.299216 & 0.000783 & 0.007576 \\
\hline & $\widehat{\gamma}$ & 0.491804 & 0.002195 & 0.004624 \\
\hline
\end{tabular}

TABLe 2: Simulation results of the L-Claim distribution for $\alpha=1.4, \gamma=0.9$, and $\sigma=1.1$.

\begin{tabular}{|c|c|c|c|c|}
\hline$n$ & Parameters & MLE & Biases & MSEs \\
\hline \multirow{3}{*}{25} & $\widehat{\alpha}$ & 1.407703 & 0.007702 & 0.089969 \\
\hline & $\widehat{\sigma}$ & 1.841798 & 0.741797 & 2.329233 \\
\hline & $\widehat{\gamma}$ & 1.817713 & 0.917713 & 2.571805 \\
\hline \multirow{3}{*}{100} & $\widehat{\alpha}$ & 1.381831 & -0.018168 & 0.027254 \\
\hline & $\widehat{\sigma}$ & 1.308675 & 0.208675 & 0.469191 \\
\hline & $\widehat{\gamma}$ & 1.237221 & 0.337221 & 0.629859 \\
\hline \multirow{3}{*}{200} & $\widehat{\alpha}$ & 1.398295 & -0.001705 & 0.012032 \\
\hline & $\widehat{\sigma}$ & 1.144440 & 0.044439 & 0.015172 \\
\hline & $\widehat{\gamma}$ & 1.051417 & 0.151417 & 0.223189 \\
\hline \multirow{3}{*}{400} & $\widehat{\alpha}$ & 1.395308 & -0.004691 & 0.006124 \\
\hline & $\widehat{\sigma}$ & 1.118567 & 0.018566 & 0.004892 \\
\hline & $\widehat{\gamma}$ & 0.956584 & 0.056584 & 0.095525 \\
\hline \multirow{3}{*}{600} & $\widehat{\alpha}$ & 1.398676 & -0.001323 & 0.004355 \\
\hline & $\widehat{\sigma}$ & 1.113499 & 0.013499 & 0.002874 \\
\hline & $\widehat{\gamma}$ & 0.951820 & 0.051820 & 0.069850 \\
\hline \multirow{3}{*}{750} & $\widehat{\alpha}$ & 1.398821 & -0.001178 & 0.003376 \\
\hline & $\widehat{\sigma}$ & 1.110305 & 0.010305 & 0.002152 \\
\hline & $\widehat{\gamma}$ & 0.934313 & 0.034313 & 0.054993 \\
\hline
\end{tabular}

Farlie-Gumbel-Morgenstern bivariate Lomax (FGMBL) distribution [23]. The word "Better" is used in the sense that the FGMBL-Claim distribution has the smallest values of the considered fitting measures; this needs to be clarified; the details are given below.

First, we estimate the unknown parameters of the fitted models using the maximum likelihood approach using the $\mathrm{R}$-script with library (BB). The estimated values of the model parameters are presented in Table 6 . To the best of our knowledge, there is no strong physical interpretation of the MLEs, and they are through an optimization technique.
Here, we use the Newton-Raphson iteration procedure using library $(\mathrm{BB})$ to estimate the model parameters and make the FGMBL-Claim distribution flexible enough to capture the information behind the data.

The comparison is done based on some discrimination measures such as the Akaike information criterion (AIC) and the Bayesian information criterion (BIC). After performing the analysis, the discrimination measures of the fitted models are presented in Table 7 . The largest values of these measures are considered the worse performance. The results provided in Table 7 reveal that the FGMBL-Claim 


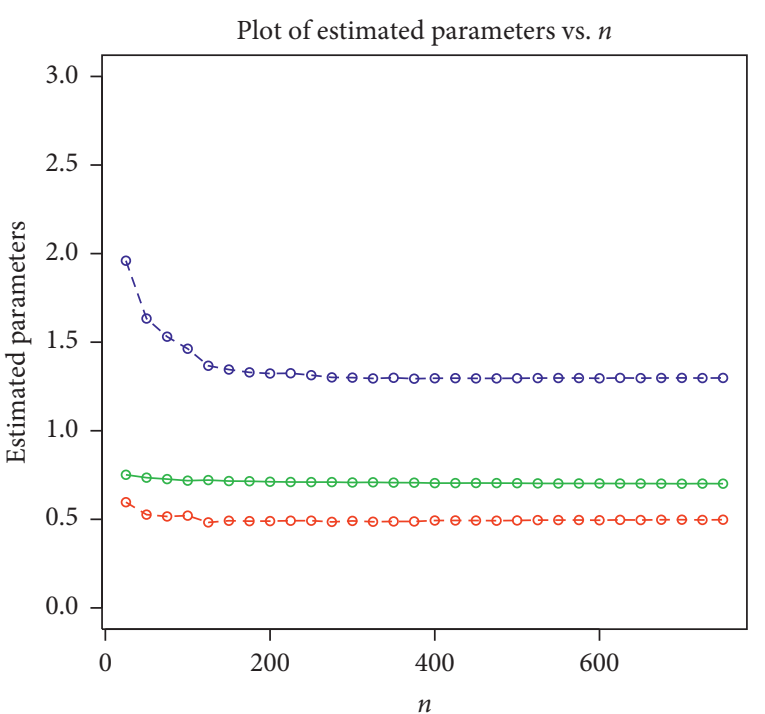

(a)

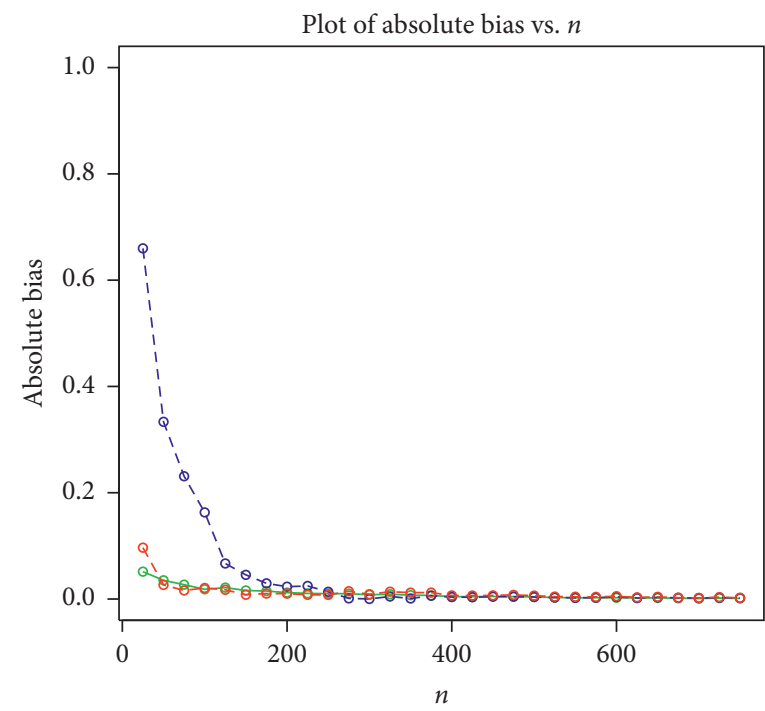

(c)

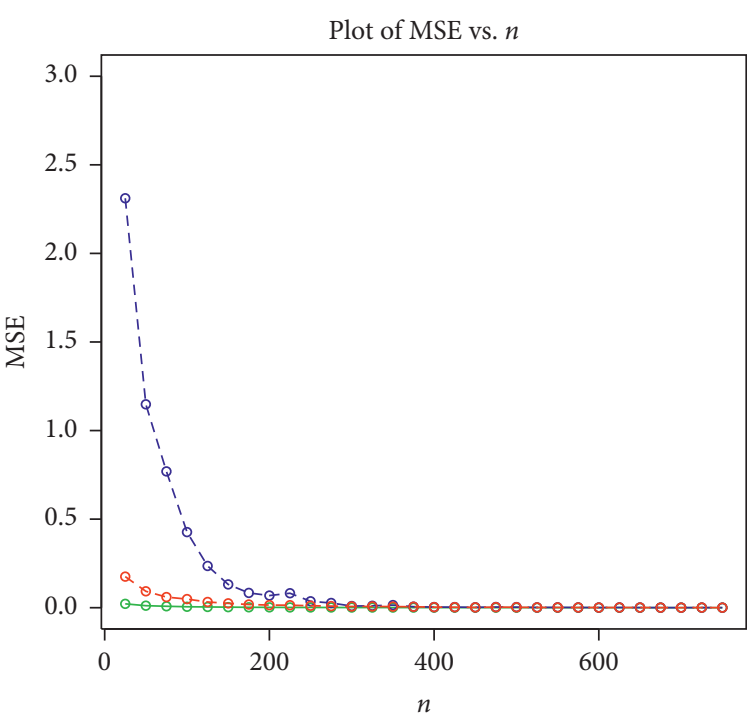

(b)

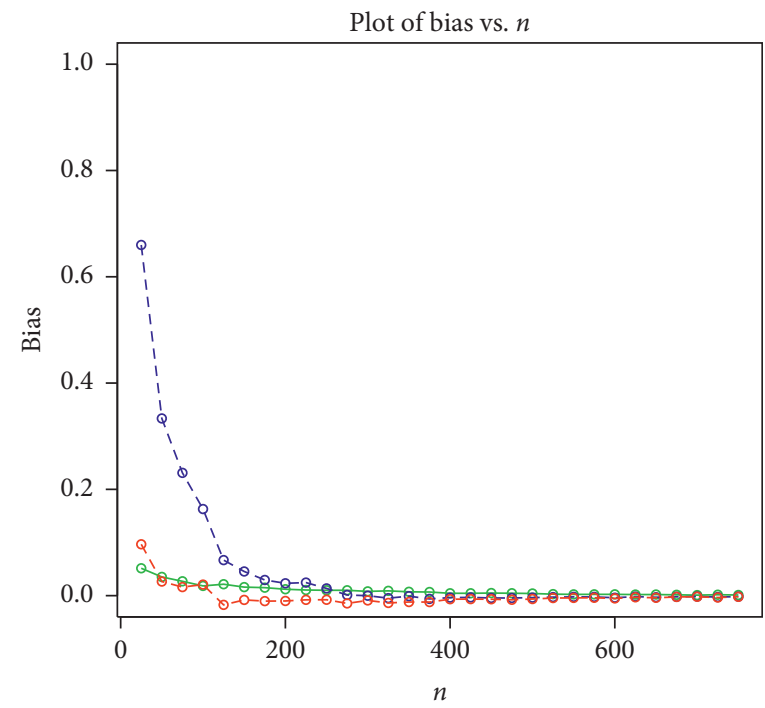

(d)

Figure 10: Plots of MLEs, MSEs, biases, and absolute biases for $\alpha=0.7$ (green - line), $\gamma=0.5$ (red - line), and $\sigma=1.3$ (blue - line).

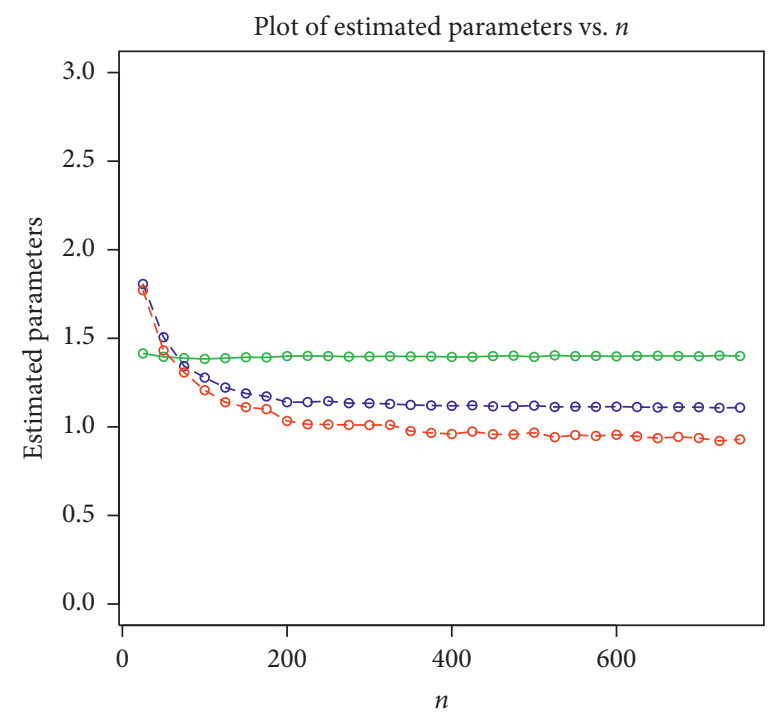

(a)

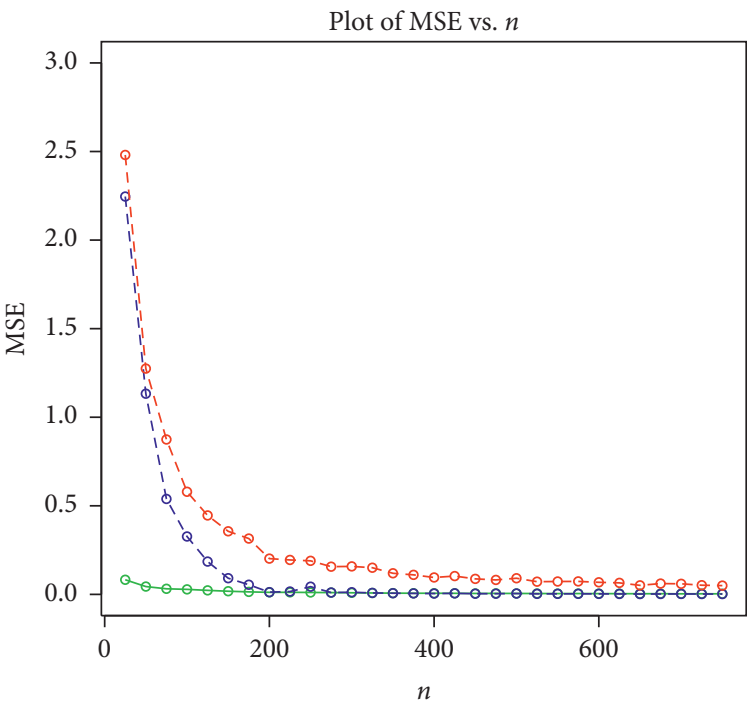

(b)

Figure 11: Continued. 


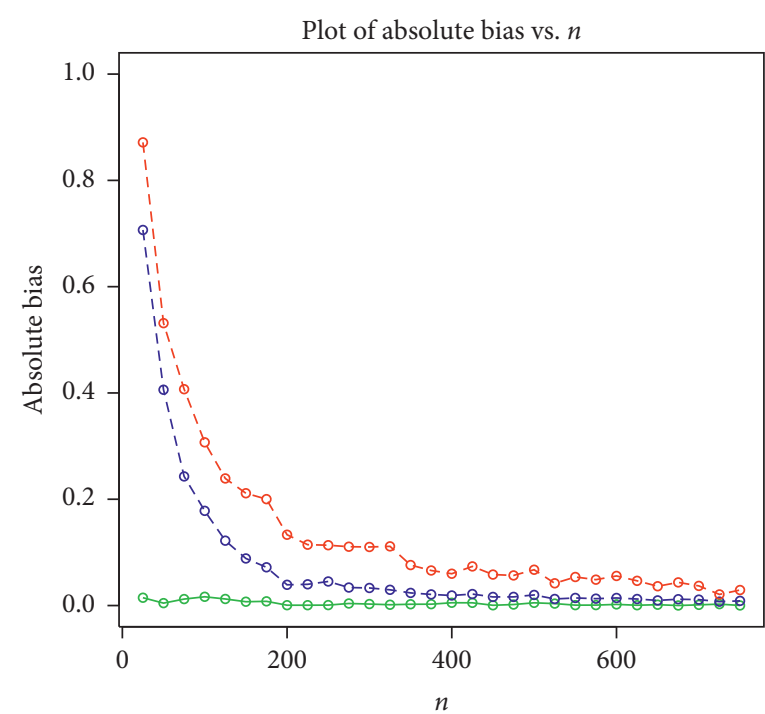

(c)

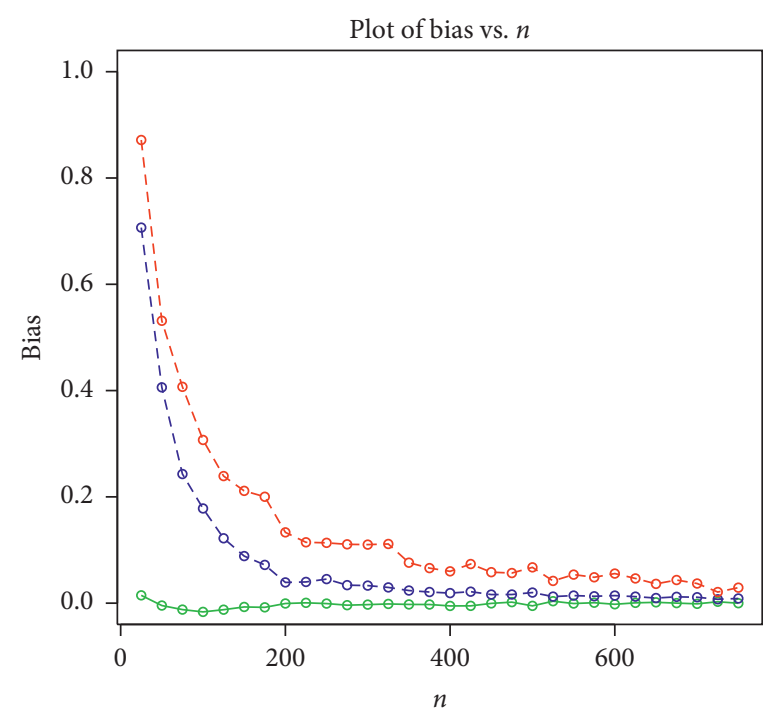

(d)

FIgURE 11: Plots of MLEs, MSEs, biases, and absolute biases for $\alpha=1.4$ (green - line), $\gamma=0.9$ (red - line), and $\sigma=1.1$ (blue - line).

TABLE 3: The estimated values of the parameters of the fitted distributions.

\begin{tabular}{|c|c|c|c|c|c|}
\hline Model & $\widehat{\alpha}$ & $\widehat{\gamma}$ & $\widehat{\sigma}$ & $\widehat{\theta}$ & $\widehat{\lambda}$ \\
\hline L-Claim & 1.879 & 0.039 & 0.017 & - & - \\
\hline Lomax & 1.845 & 0.002 & - & - & - \\
\hline T-Lomax & 1.850 & 0.874 & - & - & 0.793 \\
\hline P-Lomax & 1.732 & 0.852 & - & 31.713 & - \\
\hline E-Lomax & 1.493 & 0.006 & - & 1.882 & - \\
\hline
\end{tabular}

TABLe 4: The analytical measures of the fitted models.

\begin{tabular}{lcccc}
\hline Model & CM & AD & KS & $P$ value \\
\hline L-Claim & 0.056 & 0.320 & 0.086 & 0.951 \\
Lomax & 0.062 & 0.387 & 0.147 & 0.443 \\
T-Lomax & 0.059 & 0.374 & 0.198 & 0.407 \\
P-Lomax & 0.075 & 0.470 & 0.229 & 0.087 \\
E-Lomax & 0.108 & 0.671 & 0.150 & 0.386 \\
\hline
\end{tabular}

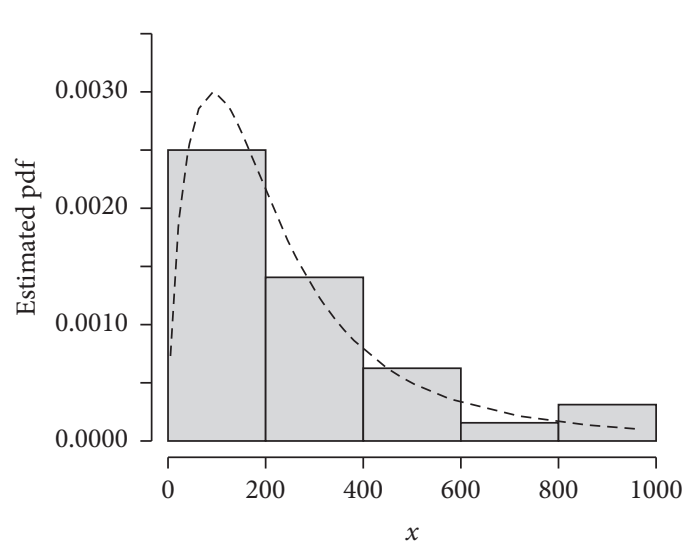

(a)

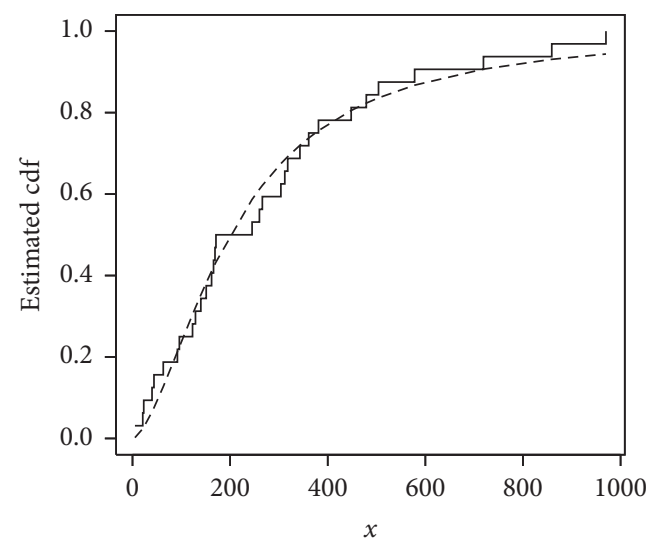

(b)

Figure 12: Continued. 


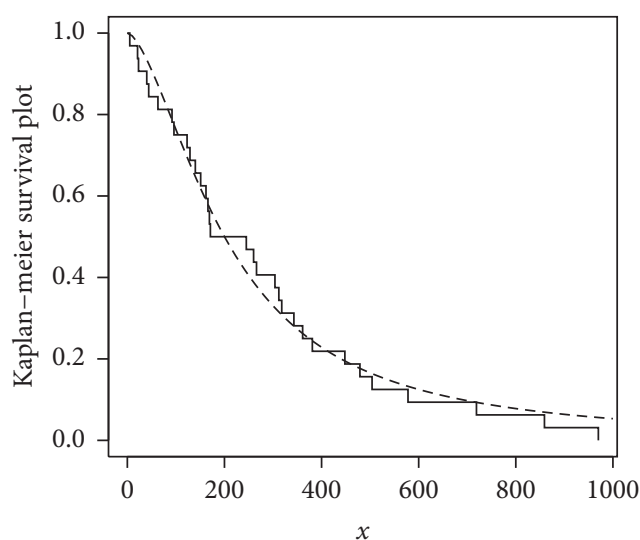

(c)

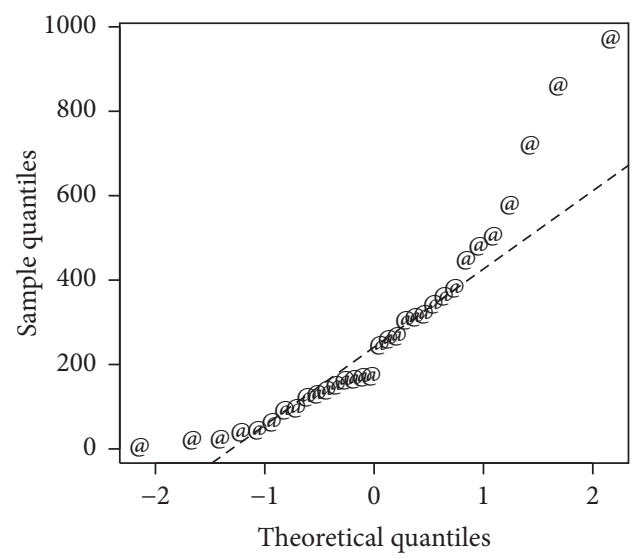

(e)

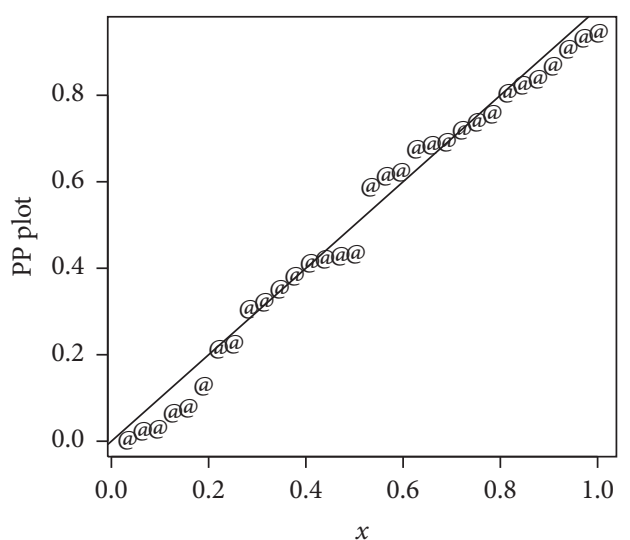

(d)

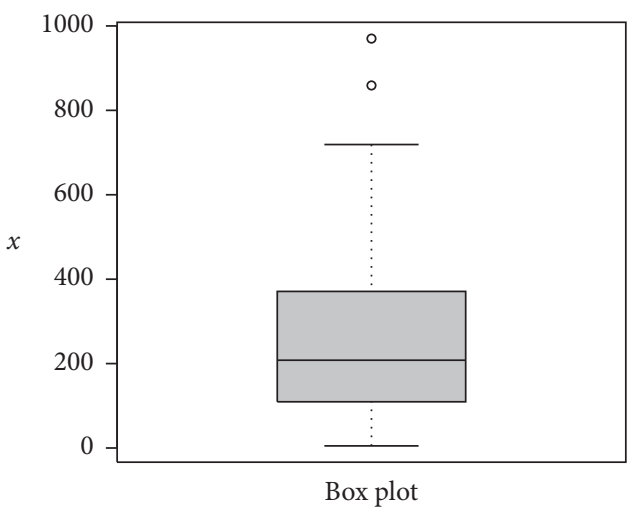

(f)

Figure 12: The fitted pdf, cdf, PP, and Kaplan-Meier survival and QQ plots of the L-Claim distribution along with the box plot of the data.

TABle 5: Summary measures of the economics data.

\begin{tabular}{lccccc}
\hline Variables & Min. & 1st qu. & Median & Mean & 3rd qu. \\
\hline$X_{1}$ & 15.070 & 19.140 & 21.170 & 23.310 & 27.610 \\
$X_{2}$ & 2.990 & 4.220 & 5.130 & 5.510 & 6.660 \\
\hline
\end{tabular}

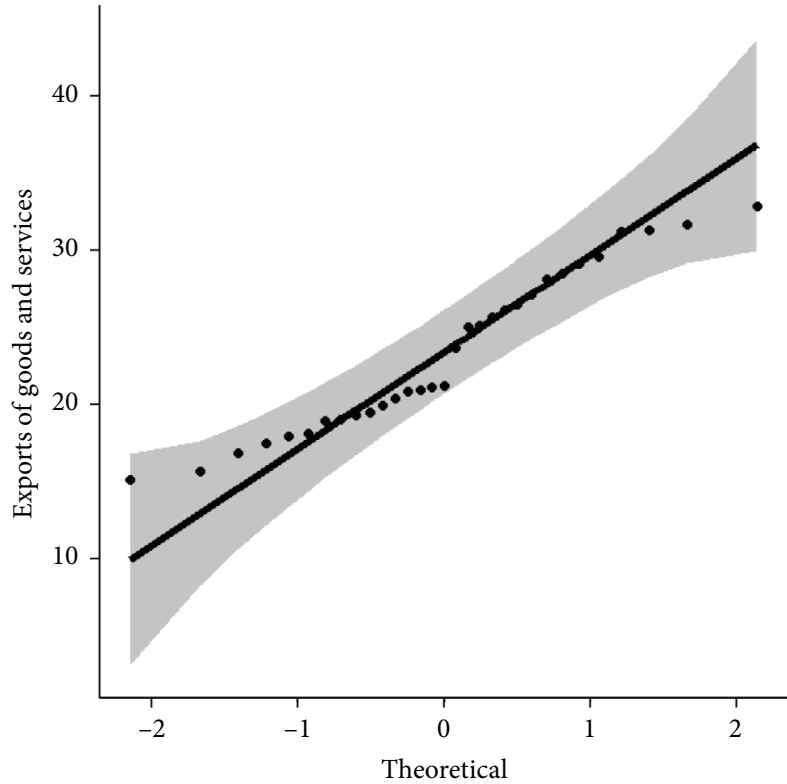

(a)

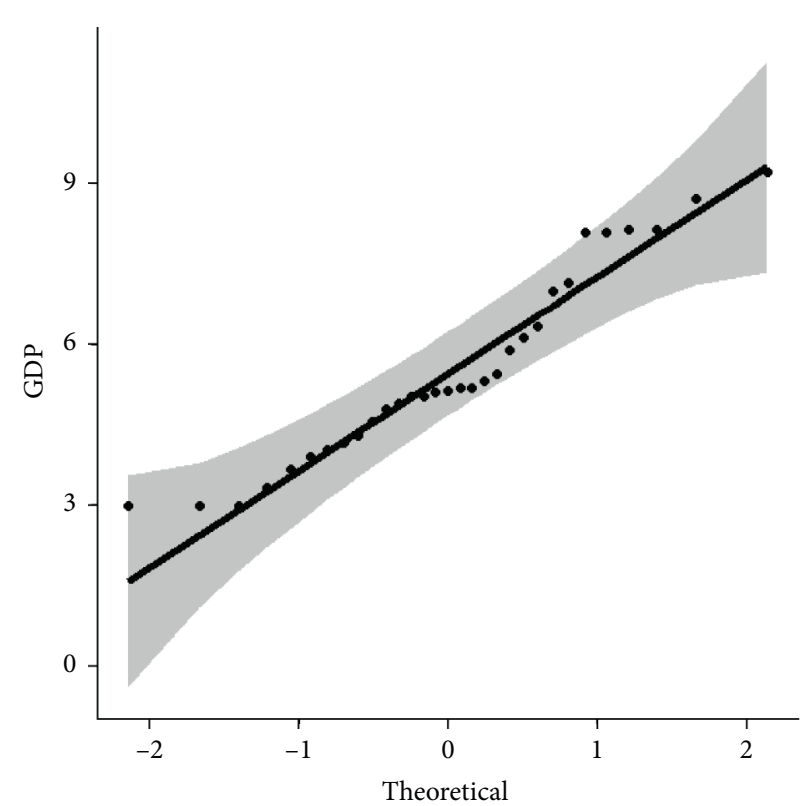

(b)

FIGURE 13: Graphical display of the QQ plot and SW test statistic result. 


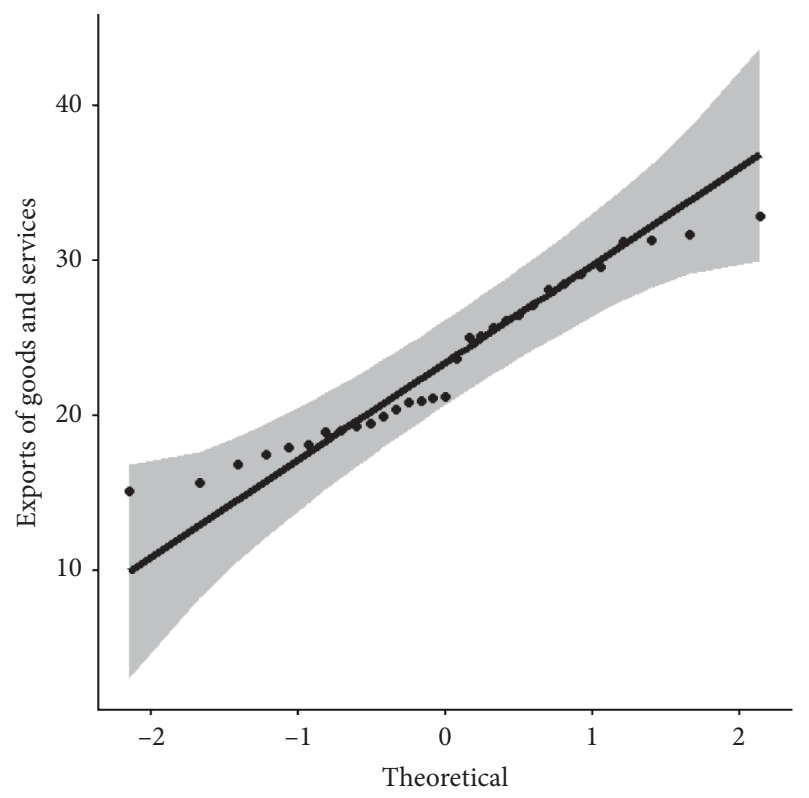

(a)

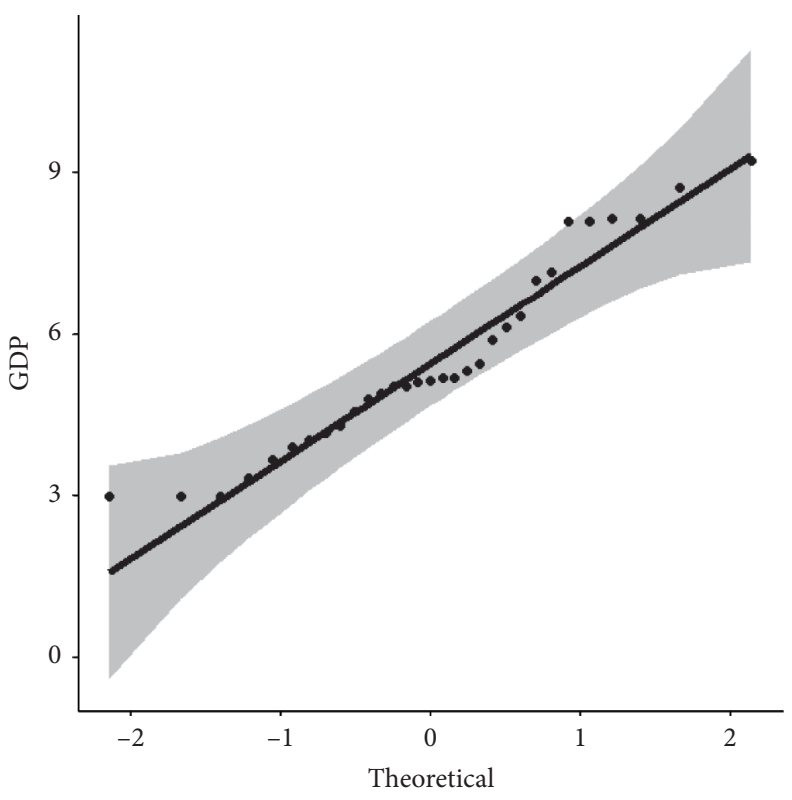

(b)

FIgURE 14: Graphical display of the QQ plot and AD test statistic result.

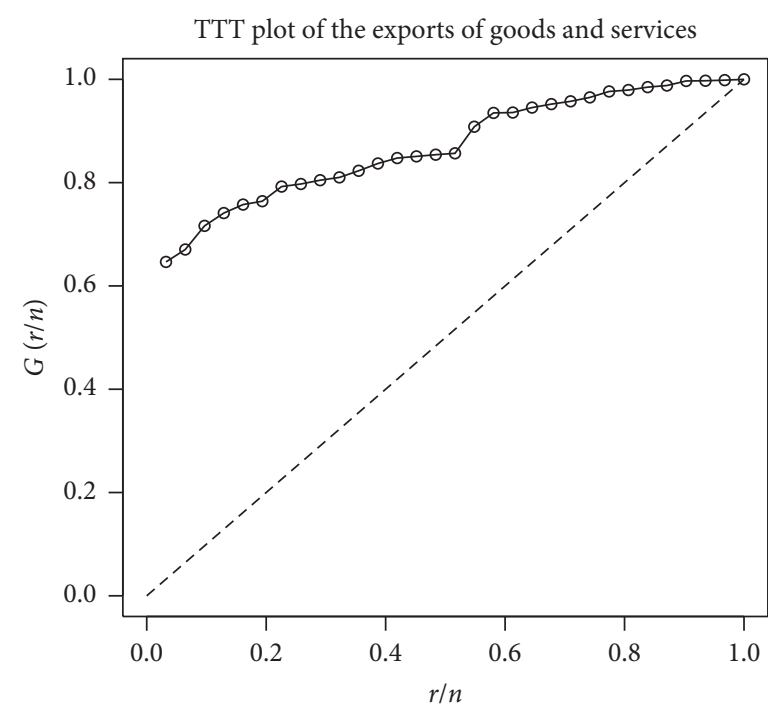

(a)

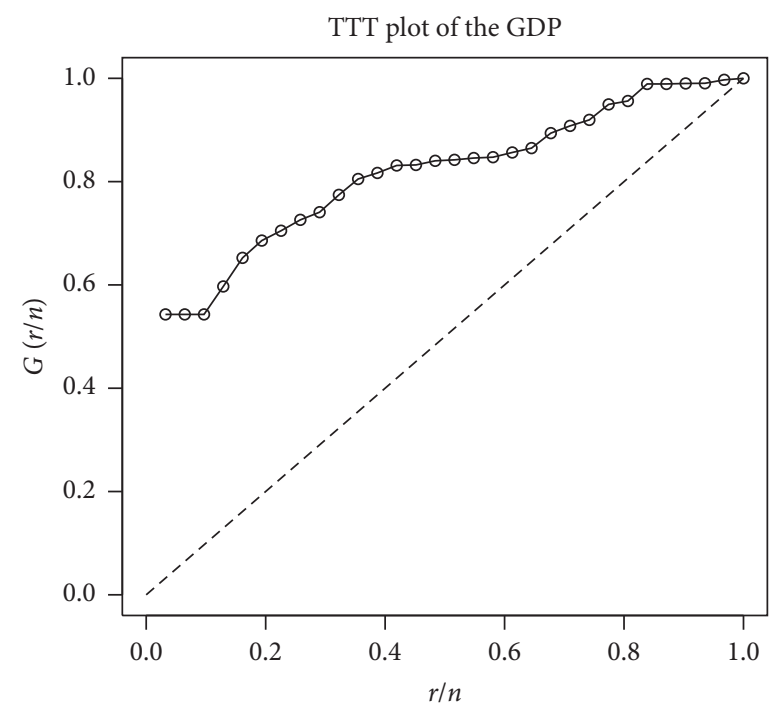

(b)

FIgure 15: The TTT plot of the economics data.

TABLE 6: MLEs of the competing models for the economics data.

\begin{tabular}{lccccccc}
\hline Fitted models & $\alpha_{1}$ & $\alpha_{2}$ & $\gamma_{1}$ & $\gamma_{2}$ & $\rho$ & $\sigma_{1}$ & $\sigma_{2}$ \\
\hline FGMBL-Claim & 1.097 & 0.909 & 1.298 & 1.170 & 0.983 & 1.306 & 1.205 \\
FGMBL & 1.256 & 1.287 & 1.696 & 1.953 & 0.854 & - & - \\
\hline
\end{tabular}

TABLE 7: Discrimination measures of the competing models for the economics data.

\begin{tabular}{lcc}
\hline Fitted models & AIC & BIC \\
\hline FGMBL-Claim & 191.656 & 194.909 \\
FGMBL & 224.549 & 229.075 \\
\hline
\end{tabular}

distribution is the best candidate model for the economics data because it has the smallest values of the fitting measures.

\section{Concluding Remarks}

In this study, a new extension of the Lomax distribution called the Lomax-Claim model is introduced. The proposed distribution is very flexible and possess heavy tails. The maximum likelihood estimators of the parameters are obtained. Furthermore, a Monte Carlo simulation study is provided to evaluate the behavior of the estimators. The proposed L-Claim model is illustrated via analyzing a 
heavy-tailed vehicle insurance loss dataset, and the comparison is made with some well-known competitive models. From the real application, we showed that the LClaim model provides a better fit to the heavy-tailed vehicle insurance loss data than the other distributions. Furthermore, a bivariate extension of the L-Claim distribution called Farlie-Gumble-Morgenstern bivariate LomaxClaim distribution is introduced. Finally, the bivariate extension of the L-Claim distribution is illustrated by analyzing economic data related to the GDP and export of goods and services.

\section{Appendix}

\section{R-code for the Analysis}

Note. In the following R-code, a is used for $\alpha, s$ is used for $\sigma, g$ is used for $\gamma$, and pm is used for the proposed model.

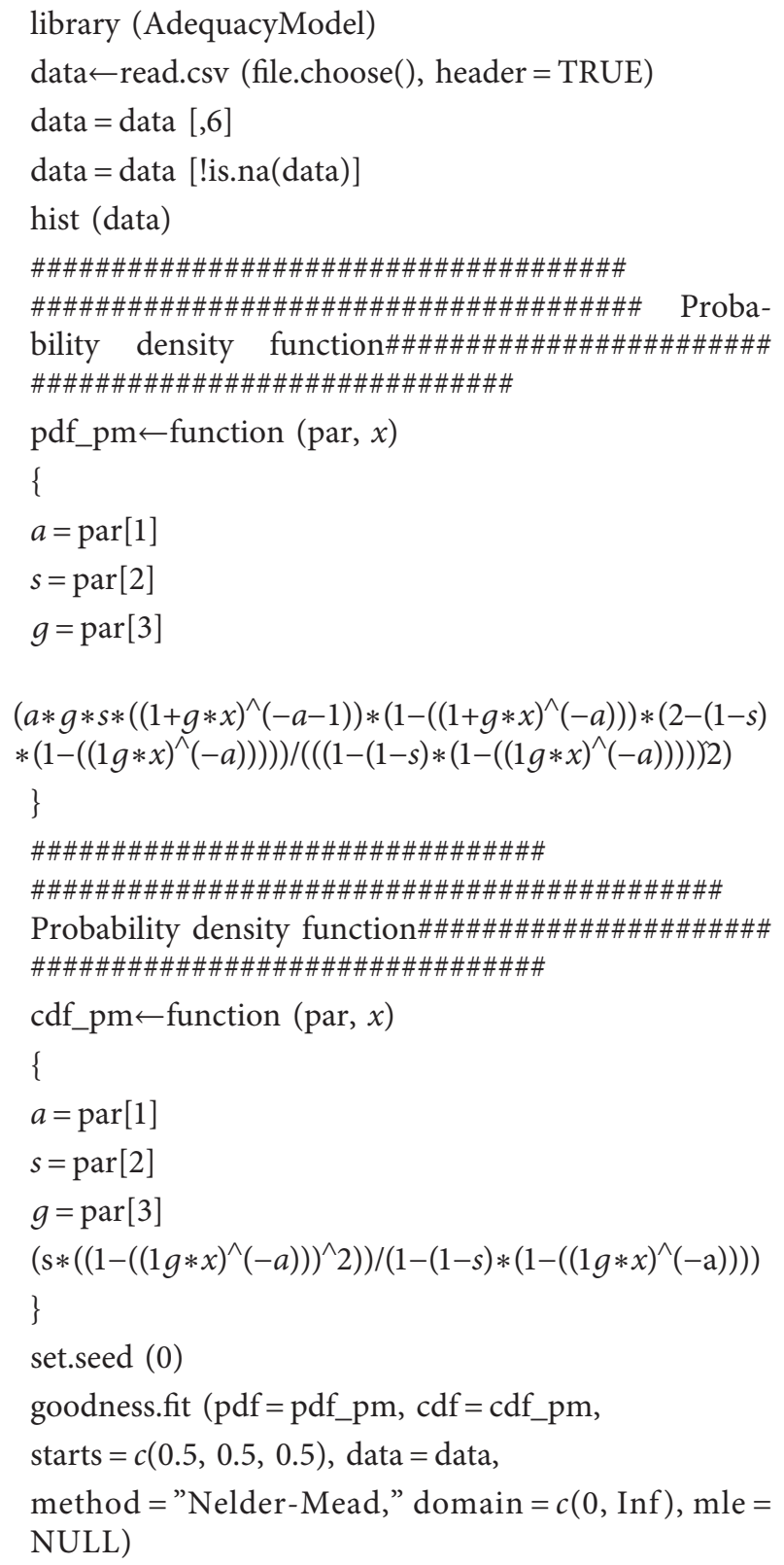

\section{Data Availability}

This work is mainly a methodological development and has been applied on secondary data, but if required, data will be provided.

\section{Conflicts of Interest}

The authors declare that they have no conflicts of interest to report regarding the present study.

\section{Acknowledgments}

The study was funded by the department of statistics, Yazd University, Yazd, Iran.

\section{References}

[1] M. H. Tahir, G. M. Cordeiro, M. Mansoor, and M. Zubair, "The weibull-lomax distribution: properties and applications," Hacettepe Journal of Mathematics and Statistics, vol. 44, no. 2, pp. 461-480, 2015.

[2] E. H. A. Rady, W. A. Hassanein, and T. A. Elhaddad, "The power lomax distribution with an application to bladder cancer data," Springer Plus, vol. 5, pp. 18-38, 2016.

[3] S. K. Ashour and M. A. Eltehiwy, "Transmuted lomax distribution," American Journal of Applied Mathematics and Statistics, vol. 1, no. 6, pp. 121-127, 2013.

[4] H. M. Salem, "The exponentiated lomax distribution: different estimation methods," American Journal of Applied Mathematics and Statistics, vol. 2, no. 6, pp. 364-368, 2014.

[5] N. M. Kilany, "Weighted lomax distribution," Springer Plus, vol. 5, no. 1, pp. 1-18, 2016.

[6] A. H. El-Bassiouny, N. F. Abdo, and H. S. Shahen, "Exponential lomax distribution," International Journal of Computer Applications, vol. 121, no. 13, pp. 24-29, 2015.

[7] G. M. Cordeiro, E. M. M. Ortega, and B. V. Popović, "The gamma-lomax distribution," Journal of Statistical Computation and Simulation, vol. 85, no. 2, pp. 305-319, 2015.

[8] B. Al-Zahrani and H. Sagor, "The poisson-lomax distribution," Revista Colombiana de Estadística, vol. 37, no. 1, pp. 225-245, 2014.

[9] A. J. Lemonte and G. M. Cordeiro, "An extended lomax distribution," Statistics, vol. 47, no. 4, pp. 800-816, 2013.

[10] M. E. Ghitany, F. A. Al-Awadhi, and L. A. Alkhalfan, "Marshall-olkin extended lomax distribution and its application to censored data," Communications in Statistics Theory and Methods, vol. 36, no. 10, pp. 1855-1866, 2007.

[11] A. S. Hassan and M. Abd-Allah, "Exponentiated weibulllomax distribution: properties and estimation," Journal of Data Science, vol. 16, no. 2, pp. 277-298, 2018.

[12] V. B. V. Nagarjuna, R. V. Vardhan, and C. Chesneau, "Kumaraswamy generalized power lomax distributionand its applications," Stats, vol. 4, no. 1, pp. 28-45, 2021.

[13] J. Mathew and C. Chesneau, "Some new contributions on the marshall-olkin Length biased lomax distribution: theory, modelling and data analysis," Mathematical and Computational Applications, vol. 25, no. 4, pp. 1-9, 2020.

[14] P. E. Oguntunde, M. A. Khaleel, M. T. Ahmed, A. O. Adejumo, and A. O. Odetunmibi, "A new generalization of the lomax distribution with increasing, decreasing, and constant failure rate," Modelling and Simulation in Engineering, vol. 2017, Article ID 6043169, 6 pages, 2017. 
[15] M. Anwar and J. Zahoor, "The half-logistic Lomax distribution for lifetime modeling," Journal of Probability and Statistics, vol. 2018, Article ID 3152807, 12 pages, 2018.

[16] M. H. Tahir, M. Adnan Hussain, G. M. Cordeiro, G. G. Hamedani, M. Mansoor, and M. Zubair, "The gumbellomax distribution: properties and applications," Journal of Statistical Theory and Applications, vol. 15, no. 1, pp. 61-79, 2016.

[17] A. Z. Afify, Z. M. Nofal, H. M. Yousof, Y. M. E. Gebaly, and N. S. Butt, "The transmuted weibull lomax distribution: properties and application," Pakistan Journal of Statistics and Operation Research, vol. 11, no. 1, pp. 135-152, 2015.

[18] S. K. Ashour and M. A. Eltehiwy, "Transmuted exponentiated lomax distribution," Australian Journal of Basic and Applied Sciences, vol. 7, no. 7, pp. 658-667, 2013.

[19] Z. Ahmad, E. Mahmoudi, and G. Hamedani, "A class of claim distributions: properties, characterizations and applications to insurance claim data," Communications in Statistics - Theory and Methods, vol. 49, pp. 1-26, 2020.

[20] D. A. Conway, "Farlie-gumbel-morgenstern distributions," in Encyclopedia of Statistical Sciences, Kotz and N. L. Johnson, Eds., pp. 28-31, Wiley, New York, NY, USA, 1983.

[21] A. L. Bowley, Elements of Statistics, Charles Scribner's Sons, New York, NY, USA, 4th edition, 1920.

[22] J. J. A. Moors, "The meaning of kurtosis: darlington reexamined," The American Statistician, vol. 40, no. 4, pp. 283-284, 1986.

[23] A. Philip and P. Y. Thomas, "On concomitants of order statistics from farlie-gumbel-morgenstern bivariate lomax distribution and its application in estimation," Journal of The Iranian Statistical Society, vol. 16, no. 2, pp. 67-95, 2017. 